\title{
Surface-enhanced Raman scattering as a higher-order Raman process
}

\author{
Niclas S. Mueller, ${ }^{1, *}$ Sebastian Heeg,,$^{1,2, \dagger}$ and Stephanie Reich ${ }^{1}$ \\ ${ }^{1}$ Freie Universität Berlin, Department of Physics, Arnimallee 14, D-14195 Berlin, Germany \\ ${ }^{2}$ School of Materials, University of Manchester, Manchester M13 9PL, United Kingdom \\ (Received 11 September 2015; revised manuscript received 29 April 2016; published 4 August 2016)
}

\begin{abstract}
We propose to understand surface-enhanced Raman scattering (SERS) as a higher-order Raman process that contains the plasmonic excitation. The SERS amplitudes are calculated with third- and fourth-order perturbation theory. Treating the plasmonic excitation as a quasiparticle, we derive analytic expressions for all coupling matrix elements. This leads to a general theory of plasmonic enhancement in SERS that can be applied to arbitrary plasmonic nanostructures. We obtain the plasmon eigenvectors of a gold nanosphere and a nanosphere dimer. They are used to calculate the enhancement of the Raman cross section of a molecule coupled to the dipole plasmon mode. The enhancement of the cross section is up to three orders of magnitude stronger than predicted by the theory of electromagnetic enhancement. The difference is most pronounced in vacuum and decreases with increasing dielectric constant of the embedding medium. The predictions from understanding SERS as a higher-order Raman process agree well with recent experiments; they highlight the dominance of plasmonic enhancement in SERS.
\end{abstract}

DOI: 10.1103/PhysRevA.94.023813

\section{INTRODUCTION}

Surface-enhanced Raman scattering (SERS) is the giant enhancement of the Raman scattering cross section for a molecule on a rough metal surface [1]. At certain hot spots the enhancement is strong enough for single-molecule detection $[2,3]$. The strong increase in sensitivity compensates the inherently weak Raman cross section of molecules and supplements the high selectivity of Raman spectroscopy [4]. This leads to applications in a variety of areas, such as analytical biochemistry, forensics, and medical diagnostics [5].

The dominant mechanism of SERS is the interaction of the Raman probe with the localized surface plasmon (LSP) of metallic nanostructures (nanoparticles, particle clusters, etc.) $[4,6]$. The theory of electromagnetic (EM) enhancement treats the local near field generated by the LSP as a strongly increased light field that drives the Raman process. Both incoming and scattered light are enhanced by a factor that is determined by the local near-field intensity, which is often abbreviated as $E^{4}$ enhancement [4,7]. In a more rigorous approach, the enhancement of the scattered light is calculated from the interaction of the radiating dipole of the Raman scatterer with the plasmonic nanostructure $[8,9]$. In backscattering this approach yields similar enhancement as the $E^{4}$-enhancement approximation [4,9], but differences occur for other scattering geometries [8].

The theory of EM enhancement is successfully employed to obtain design criteria for plasmonic nanostructures with strong SERS enhancement $[4,7]$. The predicted SERS enhancement, however, is often two to four orders of magnitude smaller than the peak $10^{7}-10^{8}$ enhancement observed experimentally [10-14]. The discrepancy was argued to arise from an additional chemical enhancement due to charge-transfer processes between molecule and metal [15]. Such chemical

\footnotetext{
*Corresponding author: niclasm@physik.fu-berlin.de

†Present address: Photonics Laboratory, ETH Zürich, 8093 Zürich, Switzerland.
}

interactions may, in fact, lead to a quenching as well as an enhancement depending on the specific experimental conditions $[4,16]$. A detailed comparison between the discrepancy of experiment and EM enhancement to the magnitude of chemical enhancement in a given configuration is therefore challenging and remains missing.

The theory of EM enhancement is a classical theory. It describes the plasmon as an external antenna for the standard Raman process. This implicitly separates a coupled quantummechanical system (plasmon and Raman scatterer) into two distinct parts. Moreover, the theory of EM enhancement does not account for the quantum nature of the plasmonic excitation. Various approaches were suggested that go beyond a classical description of SERS [17-27]. They typically focused on a quantum-mechanical description of the molecule and its coupling to the plasmonic nanostructure, but did not challenge the description of SERS by a standard Raman process.

Here we propose to understand SERS as a higher-order Raman process. The plasmonic excitation then forms an integral part of the light scattering treating plasmon and Raman probe on an equal footing. To implement our idea we adopt the microscopic theory of Raman scattering that is based on perturbation theory. SERS is described within third- and fourth-order perturbation theory. The contribution of the plasmon gives rise to an additional pair of incoming and outgoing resonances with a strong scattering intensity that we identify as the plasmonic enhancement in SERS. We obtain analytic expressions for all coupling matrix elements by employing a formalism that is based on the second quantization of the LSP. For a metallic nanosphere, we derive the LSP eigenvectors up to infinite order from Mie theory. Our theory accounts for the light and near-field polarization and leads to selection rules for the scattering processes. We calculate the plasmonic enhancement of a molecule close to a gold nanosphere and in the hot spot of a plasmonic nanodimer. It is several orders of magnitude stronger than predicted by the EM-enhancement theory, explaining the discrepancy between calculated EM enhancement and experiments. 
The article is organized as follows. In Sec. II, we derive a general theory of surface-enhanced Raman scattering for a molecule with vibronic states using higher-order perturbation theory. We first identify all relevant Raman amplitudes and give a general expression for the SERS enhancement (Sec. II A). We then employ a second quantization model for the LSP to derive the interaction Hamiltonians (Sec. II B). These are used in Sec. II C to calculate all coupling matrix elements, which leads to an explicit expression for the SERS enhancement (Sec. II D). The general theory derived in Sec. II can be applied to any plasmonic nanostructure. We demonstrate two relevant cases for SERS: In Sec. III, we calculate the SERS enhancement for a molecule coupled to the LSP of a gold nanosphere and in Sec. IV for a molecule in the gap of a gold nanoparticle dimer. Finally, in Sec. V, we compare our theory with other quantum-mechanical approaches to SERS.

\section{GENERAL THEORY}

\section{A. SERS as higher-order Raman scattering}

We consider a general SERS experiment where a plasmonic nanostructure is coupled to a molecule as a Raman scattering probe [schematically depicted in Fig. 1(a)]. In this paper, Raman scattering is described within the microscopic theory of the Raman effect that is based on perturbation theory $[28,29]$. The core idea proposed by us is to view the SERS process as higher-order Raman scattering (HORa). SERS is described as a Raman process that contains the excitation of the LSP. Our description of SERS thus differs fundamentally from the theory of EM enhancement where an ordinary Raman process is enhanced externally by the LSP. We argue that SERS should be treated as one quantum-mechanical process that must not be separated into two distinct subsystems.

We now consider the particular SERS-type scattering event schematically depicted in Fig. 1(a). An incoming photon $\omega_{\mathrm{L}}$ excites an LSP mode $w$ with energy $\hbar \omega_{w}$ [step (1) in Fig. 1(a)]. The LSP couples via its near field to the molecule and excites a transition from the molecular ground state with energy $\epsilon_{g}^{\text {vib }}$ into an intermediate vibronic state with energy $\epsilon_{j}^{\text {vib }}$ (2). This intermediate state can be a virtual state. The molecule relaxes into a final vibronic state with energy $\epsilon_{f}^{\text {vib }}$ exciting again the LSP (3). Finally, the Raman scattered light $\omega_{\mathrm{S}}$ is emitted by the LSP (4). This scattering process is depicted as a Feynman diagram in Fig. 1(b) and corresponds to fourth-order perturbation theory. The theory of EM enhancement, in contrast, treats SERS as ordinary Raman scattering, which corresponds to second-order perturbation theory [Feynman diagram in Fig. 1(c)]. Our higher-order Raman description of SERS is not to be mixed up with hyper-Raman scattering, which is described with third-order perturbation theory because two incoming photons are involved [30]. Surface-enhanced hyperRaman scattering is described with fifth-order perturbation theory by our HORa description, in contrast to a treatment with third-order perturbation theory by the EM-enhancement theory [31]. (a)

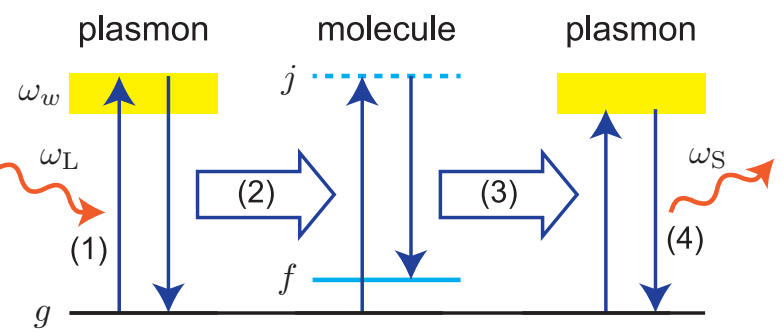

(b)

HORa description

(c)

EM theory

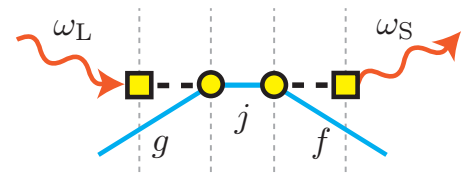

(1) (2) (3) (4)

(e)

(d)
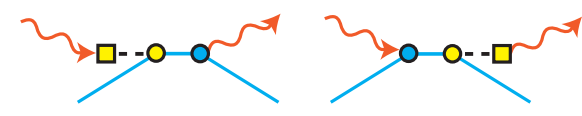

$(1,2)$

$(3,4)$

(f)

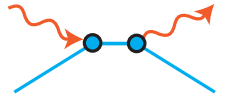

photon-vibronic

$\sim \square-\cdots$ - photon-plasmon
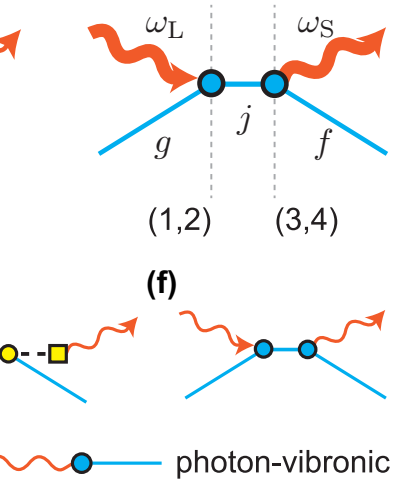

- - - - plasmon-vibronic

FIG. 1. Schematic depiction of SERS as higher-order Raman scattering (HORa). We consider a molecule with vibronic ground $g$, intermediate $j$, and final states $f$. (a) Energy diagram of the Raman process for a coupled plasmon-molecule system with the steps (1) to (4) $\left(\omega_{w}\right.$, frequency of LSP mode $w ; \omega_{\mathrm{L}}$, frequency of incoming light; $\omega_{\mathrm{S}}$, frequency of scattered light). (b) Feynman diagram of Raman process in (a), which corresponds to fourth-order perturbation theory. (c) The same Raman process described implicitly with second-order perturbation theory by the EM-enhancement theory. Enhanced incoming and scattered light are indicated by thick arrows. (d) Raman process where only incoming light and (e) only scattered light couples to the LSP. (f) Raman process without plasmonic enhancement.

In addition to the scattering process in Fig. 1(b), there are three other processes that have the same initial state (incoming photon $\omega_{\mathrm{L}}$, molecule in vibronic ground state $g$ ) and the same final state (Raman scattered photon $\omega_{\mathrm{S}}$, molecule in a final vibronic state $f$ ). They are the two processes where either incoming or scattered light couples to the LSP [Figs. 1(d) and 1(e)] and the Raman process without plasmonic enhancement [Fig. 1(f)]. The scattering pathways in Figs. 1(b), and 1(d)-1(f) interfere, leading to the selective increase or decrease of plasmonic enhancement at a given excitation wavelength. This interference has to be distinguished from interferences between electronic and vibronic Raman scattering that might lead to Fano line shapes [32,33].

We now calculate the Raman scattering amplitudes represented by the Feynman diagrams in Fig. 1 within perturbation theory [28,29];

$$
K_{\mathrm{pl}-\mathrm{pl}}^{w, w^{\prime}, j}\left(\omega_{\mathrm{L}}\right)=\frac{\mathcal{M}_{\mathrm{pt}-\mathrm{pl}}^{w^{\prime}} \mathcal{M}_{\mathrm{pl}-\mathrm{vib}}^{w^{\prime}, j} \mathcal{M}_{\mathrm{vib}-\mathrm{pl}}^{w, j} \mathcal{M}_{\mathrm{pl}-\mathrm{pt}}^{w}}{\left(\hbar \omega_{\mathrm{L}}+\epsilon_{g}^{\mathrm{vib}}-\epsilon_{f}^{\mathrm{vib}}-\hbar \omega_{w^{\prime}}-i \gamma_{w^{\prime}}\right)\left(\hbar \omega_{\mathrm{L}}+\epsilon_{g}^{\mathrm{vib}}-\epsilon_{j}^{\mathrm{vib}}-i \gamma_{j}^{\mathrm{vib}}\right)\left(\hbar \omega_{\mathrm{L}}+\epsilon_{g}^{\mathrm{vib}}-\hbar \omega_{w}-i \gamma_{w}\right)}
$$


is the scattering amplitude that corresponds to Fig. 1(b),

$$
K_{\mathrm{pl}-\mathrm{pt}}^{w, j}\left(\omega_{\mathrm{L}}\right)=\frac{\mathcal{M}_{\mathrm{pt}-\mathrm{vib}}^{j} \mathcal{M}_{\mathrm{vib}-\mathrm{pl}}^{w, j} \mathcal{M}_{\mathrm{pl}-\mathrm{pt}}^{w}}{\left(\hbar \omega_{\mathrm{L}}+\epsilon_{g}^{\mathrm{vib}}-\epsilon_{j}^{\mathrm{vib}}-i \gamma_{j}^{\mathrm{vib}}\right)\left(\hbar \omega_{\mathrm{L}}+\epsilon_{g}^{\mathrm{vib}}-\hbar \omega_{w}-i \gamma_{w}\right)}
$$

corresponds to Fig. 1(d),

$$
K_{\mathrm{pt-pl}}^{w, j}\left(\omega_{\mathrm{L}}\right)=\frac{\mathcal{M}_{\mathrm{pt-pl}}^{w} \mathcal{M}_{\mathrm{pl}-\mathrm{vib}}^{w, j} \mathcal{M}_{\mathrm{vib}-\mathrm{pt}}^{j}}{\left(\hbar \omega_{\mathrm{L}}+\epsilon_{g}^{\mathrm{vib}}-\epsilon_{f}^{\mathrm{vib}}-\hbar \omega_{w}-i \gamma_{w}\right)\left(\hbar \omega_{\mathrm{L}}+\epsilon_{g}^{\mathrm{vib}}-\epsilon_{j}^{\mathrm{vib}}-i \gamma_{j}^{\mathrm{vib}}\right)}
$$

corresponds to Fig. 1(e), and

$$
K_{\mathrm{pt-pt}}^{j}\left(\omega_{\mathrm{L}}\right)=\frac{\mathcal{M}_{\mathrm{pt}-\mathrm{vib}}^{j} \mathcal{M}_{\mathrm{vib}-\mathrm{pt}}^{j}}{\hbar \omega_{\mathrm{L}}+\epsilon_{g}^{\mathrm{vib}}-\epsilon_{j}^{\mathrm{vib}}-i \gamma_{j}^{\text {vib }}}
$$

corresponds to Fig. 1(f). $\gamma_{w}=\hbar / 2 \tau_{w}$ is the inverse of the lifetime $\tau_{w}$ of an LSP mode with frequency $\omega_{w} \cdot \gamma_{j}^{\text {vib }}$ is the inverse of the lifetime of the intermediate vibronic state $j$. $\mathcal{M}_{k-l}$ are the coupling matrix elements that correspond to the vertices in the Feynman diagrams; they are discussed in detail below.

The excitation-energy dependence of the Raman scattering cross section is generated by the energy terms in the denominators of Eqs. (1)-(4). We obtain two resonances that are induced by plasmonic excitations in the SERS Feynman diagrams. If the energy $\hbar \omega_{\mathrm{L}}$ of the incoming light matches the energy $\hbar \omega_{w}$ of an LSP mode $w$, the real part of an energy term vanishes, generating an incoming resonance with a spectral width $2 \gamma_{w}$. Similarly, an outgoing resonance occurs if $\hbar \omega_{\mathrm{S}}$ matches an LSP mode. These LSP-mediated resonances are equivalent to the intrinsic Raman resonances with a molecular transition [28,29]. Comparing the LSP resonances obtained from the HORa description in Eqs. (1)-(3) with the enhancement predicted by the theory of EM enhancement, we note that they will occur at similar energy as the maximum in the EM enhancement, but their intensity and excitation-energy dependence will be very different (see below).

The experimentally accessible plasmonic enhancement factor of the Raman cross section is calculated as

$$
\operatorname{EF}\left(\omega_{\mathrm{L}}\right)=\frac{\left|\sum_{w, w^{\prime}, j} K_{\mathrm{tot}}^{w, w^{\prime}, j}\left(\omega_{\mathrm{L}}\right)\right|^{2}}{\left|\sum_{j} K_{\mathrm{ptpt}}^{j}\left(\omega_{\mathrm{L}}\right)\right|^{2}},
$$

with $\quad K_{\mathrm{tot}}^{w, w^{\prime}, j}=K_{\mathrm{pl}-\mathrm{pl}}^{w, j}+K_{\mathrm{pl}-\mathrm{pt}}^{w, j}+K_{\mathrm{pt}-\mathrm{pl}}^{w, j}+K_{\mathrm{pt}-\mathrm{pt}}^{j} \quad$ from Eqs. (1)-(4). The four scattering processes in Fig. 1 have the same initial and final states. They are therefore summed over all intermediate vibronic states $j$ and LSP modes $w, w^{\prime}$ in Eq. (5), before calculating the intensity of the scattered light as the absolute square of the scattering amplitudes. This is important, because scattering processes with amplitudes of opposite sign (or phase) diminish by destructive quantum interference.

\section{B. Interaction Hamiltonians}

We derived the general framework to describe SERS as higher-order Raman scattering within perturbation theory. The main ingredients to calculate the plasmonic enhancement in Eq. (5) are the coupling matrix elements $\mathcal{M}_{k-l}=\left\langle k\left|\hat{\mathcal{H}}_{\text {int }}\right| l\right\rangle$. They are composed of an interaction Hamiltonian $\mathcal{H}_{\text {int }}$ and wave functions $|l\rangle,|k\rangle$ of the intermediate states before and after the interaction. The matrix elements also allow analyzing SERS selection rules by considering the symmetries of initial and final state and their interaction within group theory [29]. Our theory accounts for the polarization of the plasmonic near field and the orientation of the molecular transition dipole. Including other aspects of SERS, such as charge-transfer transitions between metal and molecule or the field gradient effect, will be the subject of future work [34,35].

We now derive expressions for the interaction Hamiltonians. To describe the coupling mechanisms that involve the LSP, we use second quantization as suggested by Finazzi and Ciccacci [36]. This formalism is also based on perturbation theory and is employed to calculate all matrix elements in an intuitive way. We consider the weak-coupling regime where the plasmonic and probe system retain their electronic properties. We stress that the general framework introduced in Sec. II A is also applicable to the strong-coupling regime by using alternative approaches for calculating the matrix elements (see, e.g., Ref. [37]). The plasmonic eigenmodes $w$ are treated as a harmonic oscillator with the internal Hamiltonian

$$
\hat{\mathcal{H}}_{\text {internal }}=\frac{\mathcal{N} V_{\mathrm{p}}}{2} \sum_{w}\left(\frac{1}{m} \hat{\Pi}_{w}^{2}+m \omega_{w}^{2} \hat{\Psi}_{w}^{2}\right),
$$

where $\mathcal{N}$ is the density of free electrons, $V_{\mathrm{p}}$ the volume of the plasmonic nanostructure, and $m$ the electron mass, and

$$
\hat{\Psi}_{w}=\sqrt{\frac{\hbar}{2 m \mathcal{N} V_{\mathrm{p}} \omega_{w}}}\left(\hat{a}_{w}^{\dagger}+\hat{a}_{w}\right)
$$

and

$$
\hat{\Pi}_{w}=i \sqrt{\frac{m \hbar \omega_{w}}{2 \mathcal{N} V_{\mathrm{p}}}}\left(\hat{a}_{w}^{\dagger}-\hat{a}_{w}\right)
$$

are generalized position and momentum coordinates that contain plasmonic creation and annihilation operators $\hat{a}_{w}^{\dagger}$ and $\hat{a}_{w}$, respectively. The internal Hamiltonian is written in the form of Eq. (6) by ignoring losses initially. Decay is instead accounted for in the denominators of the Raman scattering amplitudes [Eqs. (1)-(3)] by the inverse plasmon lifetime $\gamma_{w}$, as is common practice within the microscopic theory of Raman scattering $[28,29]$.

The external light field is described in second quantization by the vector potential $[38,39]$

$$
\hat{\mathbf{A}}_{\mathrm{pt}}(\mathbf{r})=\tilde{A}_{\mathrm{pt}} \sum_{\mathbf{k}_{\mathrm{pt}}, S} \boldsymbol{\varepsilon}_{\mathbf{k}_{\mathrm{p} t}, s}\left(\hat{a}_{\mathbf{k}_{\mathrm{pt}}, s} e^{i \mathbf{k}_{\mathrm{pt}} \cdot \mathbf{r}}+\hat{a}_{\mathbf{k}_{\mathrm{p} t}, s}^{\dagger} e^{-i \mathbf{k}_{\mathrm{pt}} \cdot \mathbf{r}}\right),
$$




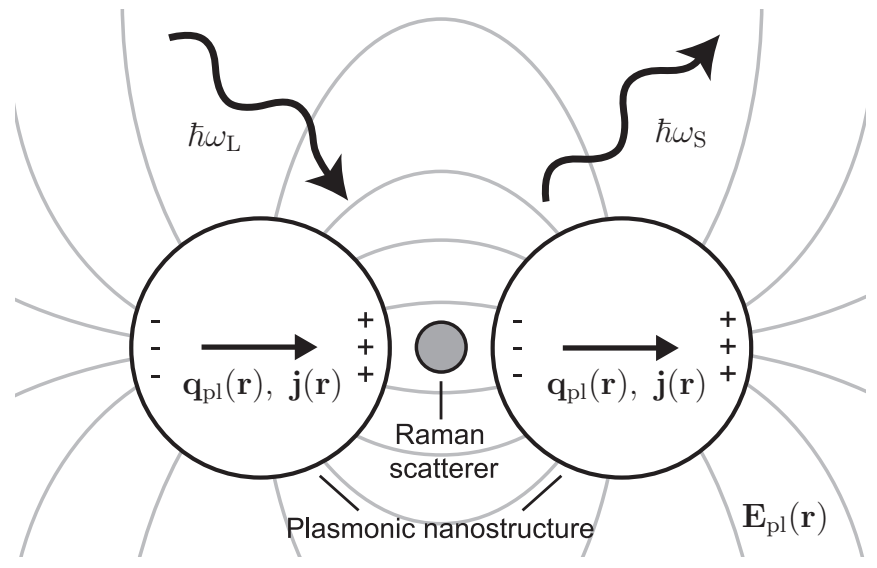

FIG. 2. Schematic SERS system. The Raman scatterer (gray disk) is coupled to the dipole mode (here with index "pl") of a plasmonic nanoparticle dimer (white disks). The gray lines illustrate the near field generated by the dipole plasmon mode $\mathbf{E}_{\mathrm{pl}}(\mathbf{r})$ with polarization along the field lines. $+/-$ signs indicate the dipole moment induced by the LSP dipole mode.

with wave vectors $\mathbf{k}_{\mathrm{pt}}$ and polarizations $\boldsymbol{\varepsilon}_{\mathbf{k}_{\mathrm{pt}}, s}$. $\tilde{A}_{\mathrm{pt}}=$ $\sqrt{\hbar / 2 \omega_{\mathrm{pt}} V_{\mathrm{R}} \epsilon_{0} \epsilon_{\mathrm{m}}}$, where $V_{\mathrm{R}}$ is a volume that contains the external light field, $\omega_{\mathrm{pt}}$ is the frequency of the light field, and $\epsilon_{\mathrm{m}}$ is the dielectric constant of the surrounding medium. Harmonic time dependence is assumed throughout.

To derive the plasmon-photon interaction Hamiltonian, the external light field is included into this description as a generalized coordinate,

$$
\hat{A}_{w}=\frac{1}{\sqrt{V_{\mathrm{p}}}} \int_{V_{\mathrm{p}}} d V^{\prime} \hat{\mathbf{A}}_{\mathrm{pt}}\left(\mathbf{r}^{\prime}, \omega_{w}\right) \cdot \mathbf{q}_{w}\left(\mathbf{r}^{\prime}\right),
$$

by a projection of $\hat{\mathbf{A}}_{\mathrm{pt}}(\mathbf{r})$ onto the eigenvector $\mathbf{q}_{w}(\mathbf{r})$ of an LSP mode $w$ [36]. The eigenvectors are normalized as

$$
\sum_{w} \mathbf{q}_{w}(\mathbf{r}) \cdot \mathbf{q}_{w}\left(\mathbf{r}^{\prime}\right)=\delta\left(\mathbf{r}-\mathbf{r}^{\prime}\right) .
$$

$\hat{\mathcal{H}}_{\text {internal }}$ is modified with a Peierl's substitution $\hat{\Pi}_{w} \rightarrow \hat{\Pi}_{w}+$ $e \hat{A}_{w}$, which leads to a plasmon-photon interaction Hamiltonian,

$$
\hat{\mathcal{H}}_{\mathrm{pl}-\mathrm{pt}}=\frac{e \mathcal{N} V_{\mathrm{p}}}{m} \sum_{w} \hat{\Pi}_{w} \hat{A}_{w}
$$

We now consider the excitation of a vibronic transition in the molecule by the LSP. The molecule interacts with the LSP via the electric field $\mathbf{E}_{\mathrm{LSP}}(\mathbf{r})$ generated by the plasmonic nanostructure, as shown schematically in Fig. 2 (the index "LSP" refers to the contribution of all excited plasmon modes). As an interaction Hamiltonian we use $\hat{\mathcal{H}}_{\text {pl-vib }}=-\hat{\boldsymbol{\mu}} \cdot \hat{\mathbf{E}}_{\mathrm{LSP}}(\mathbf{r})$ with the dipole operator of the molecule $\hat{\boldsymbol{\mu}}=\sum_{m, n} \boldsymbol{\mu}_{m n}|n\rangle\langle m|$ [40]. $\boldsymbol{\mu}_{m n}$ is a matrix element that corresponds to the transition dipole moment of a transition from vibronic state $|m\rangle$ to state $|n\rangle$. The electric field

$$
\mathbf{E}_{\mathrm{LSP}}(\mathbf{r})=i \omega \mu_{0} \int_{V_{\mathrm{p}}} d V^{\prime} \stackrel{\leftrightarrow}{\mathbf{G}}\left(\mathbf{r}, \mathbf{r}^{\prime}\right) \mathbf{j}\left(\mathbf{r}^{\prime}\right)
$$

is created by the electrical current density $\mathbf{j}(\mathbf{r})$ in the plasmonic nanostructure (see Fig. 2); $\stackrel{\leftrightarrow}{\mathbf{G}}\left(\mathbf{r}, \mathbf{r}^{\prime}\right.$ ) is the dyadic Green's function [41], Eq. (B4). We quantize the LSP and replace j(r) with the generalized momentum operator [36]

$$
\hat{\Pi}_{w}=\frac{m}{e \mathcal{N} \sqrt{V_{\mathrm{p}}}} \int_{V_{\mathrm{p}}} d V^{\prime} \hat{\mathbf{j}}\left(\mathbf{r}^{\prime}\right) \cdot \mathbf{q}_{w}\left(\mathbf{r}^{\prime}\right) .
$$

This leads to a quantized electric field

$$
\hat{\mathbf{E}}_{\mathrm{LSP}}(\mathbf{r})=\frac{i \mu_{0} e \mathcal{N} V_{\mathrm{p}}}{m} \sum_{w} \omega_{w} \hat{\Pi}_{w} \mathbf{G}_{w}(\mathbf{r}),
$$

with

$$
\mathbf{G}_{w}(\mathbf{r})=\frac{1}{\sqrt{V_{\mathrm{p}}}} \int_{V_{\mathrm{p}}} d V^{\prime} \stackrel{\leftrightarrow}{\mathbf{G}}\left(\mathbf{r}, \mathbf{r}^{\prime}\right) \mathbf{q}_{w}\left(\mathbf{r}^{\prime}\right)
$$

The remaining Hamiltonian for coupling between the external light field and a molecule is $\hat{\mathcal{H}}_{\mathrm{pt}-\mathrm{vib}}=-\hat{\boldsymbol{\mu}} \cdot \hat{\mathbf{E}}_{\mathrm{pt}}(\mathbf{r})$, where $\hat{\mathbf{E}}_{\mathrm{pt}}=$ $-\partial \hat{\mathbf{A}}_{\mathrm{pt}} / \partial t$.

\section{Matrix elements}

The interaction Hamiltonians $\hat{\mathcal{H}}_{\text {pl-pt }}, \hat{\mathcal{H}}_{\text {pl-vib }}$, and $\hat{\mathcal{H}}_{\text {pt-vib }}$ allow calculating all matrix elements in Eqs. (1)-(4). For the plasmon-photon coupling matrix elements we obtain

$$
\mathcal{M}_{\mathrm{pl}-\mathrm{pt}}^{w}=\left\langle 1_{w}^{\mathrm{pl}}, 0^{\mathrm{pt}}\left|\hat{\mathcal{H}}_{\mathrm{pl}-\mathrm{pt}}\right| 0_{w}^{\mathrm{pl}}, 1_{\mathbf{k}_{\mathrm{pt}}, \varepsilon_{\mathrm{pt}}}^{\mathrm{pt}}\right\rangle=i e \hbar \sqrt{\frac{\mathcal{N} V_{\mathrm{p}}}{4 m V_{\mathrm{R}} \epsilon_{0} \epsilon_{m}}} \varepsilon_{w}^{\mathrm{pt}}
$$

and $\mathcal{M}_{\mathrm{pt}-\mathrm{pl}}^{w}=\left(\mathcal{M}_{\mathrm{pl}-\mathrm{pt}}^{w}\right)^{*}$, where we set $\omega_{\mathrm{pt}}=\omega_{\mathrm{pl}}$ and

$$
\varepsilon_{w}^{\mathrm{pt}}=\frac{1}{\sqrt{V_{\mathrm{p}}}} \int_{V_{\mathrm{p}}} d V^{\prime} \boldsymbol{\varepsilon}_{\mathrm{pt}} \cdot \mathbf{q}_{w}\left(\mathbf{r}^{\prime}\right) e^{i \mathbf{k}_{\mathrm{pt}} \cdot \mathbf{r}^{\prime}} .
$$

Explicit expressions for the plasmon-molecule coupling matrix elements are

$$
\begin{aligned}
\mathcal{M}_{\mathrm{vib}-\mathrm{pl}}^{w, i} & =\left\langle 0_{w}^{\mathrm{pl}}, i^{\mathrm{vib}}\left|\hat{\mathcal{H}}_{\mathrm{pl}-\mathrm{vib}}\right| 1_{w}^{\mathrm{pl}}, g^{\mathrm{vib}}\right\rangle \\
& =\mu_{0} e \sqrt{\frac{\hbar \mathcal{N} V_{\mathrm{p}} \omega_{w}^{3}}{2 m}} \boldsymbol{\mu}_{g j} \cdot \mathbf{G}_{w}(\mathbf{r})
\end{aligned}
$$

and with the assumption that $\boldsymbol{\mu}_{g j} \| \boldsymbol{\mu}_{j f}$ we obtain $\mathcal{M}_{\mathrm{pl} \text {-vib }}^{w, j}=$ $-\mu_{j f} / \mu_{g j} \mathcal{M}_{\mathrm{vib}-\mathrm{pl}}^{w, j}$. Finally, the matrix elements for lightmolecule coupling are

$$
\begin{aligned}
\mathcal{M}_{\mathrm{vib}-\mathrm{pt}}^{j} & =\left\langle 0^{\mathrm{pt}}, j^{\mathrm{vib}}\left|\hat{\mathcal{H}}_{\mathrm{pt}-\mathrm{vib}}\right| 1_{\mathbf{k}_{\mathrm{pt}}, \boldsymbol{\varepsilon}_{\mathrm{pt}}}^{\mathrm{pt}}, g^{\mathrm{vib}}\right\rangle \\
& =-i \sqrt{\frac{\hbar \omega_{\mathrm{pt}}}{2 V_{\mathrm{R}} \epsilon_{0} \epsilon_{m}}} e^{i \mathbf{k}_{\mathrm{pt}} \cdot \mathbf{r}} \boldsymbol{\varepsilon}_{\mathrm{pt}} \cdot \boldsymbol{\mu}_{g j}
\end{aligned}
$$

and $\mathcal{M}_{\mathrm{pt-vib}}^{j}=\mu_{j f} / \mu_{g j}\left(\mathcal{M}_{\mathrm{vib}-\mathrm{pt}}^{j}\right)^{*}$ if $\boldsymbol{\mu}_{g j} \| \boldsymbol{\mu}_{j f}$.

\section{Explicit expression for SERS enhancement}

We have now all tools at hand to obtain an explicit expression for the SERS enhancement from Eq. (5). We consider one dominant plasmon mode $w$, but the theory can be extended to multiple plasmonic modes by summing over the excitations. To derive expressions for the coupling 
matrix elements in Eqs. (1)-(4), we made the assumption that molecule-metal interactions can be described in the weak-coupling regime with a perturbative approach, i.e., the electronic properties of plasmon and molecule are not affected by their interaction. Both the external light field and the LSP were described with second quantization. The properties of the molecule were included by its transition dipole moment. SERS enhancement is then calculated as

$$
\mathrm{EF}\left(\omega_{\mathrm{L}}\right)=\left|1+\frac{\tilde{\mathcal{M}}_{1} \tilde{\mathcal{M}}_{2}}{\left(\hbar \omega_{\mathrm{L}}-\epsilon_{\mathrm{f}}^{\mathrm{vib}}-\hbar \omega_{w}-i \gamma_{w}\right)\left(\hbar \omega_{\mathrm{L}}-\hbar \omega_{w}-i \gamma_{w}\right)}+\frac{\tilde{\mathcal{M}}_{1}}{\hbar \omega_{\mathrm{L}}-\hbar \omega_{w}-i \gamma_{w}}+\frac{\tilde{\mathcal{M}}_{2}}{\hbar \omega_{\mathrm{L}}-\epsilon_{\mathrm{f}}^{\mathrm{vib}}-\hbar \omega_{w}-i \gamma_{w}}\right|^{2}
$$

with the coupling factors

$$
\tilde{\mathcal{M}}_{1}=-\varepsilon_{w}^{\mathrm{pt}} e^{-i \mathbf{k}_{\mathrm{pt}} \cdot \mathbf{r}} \tilde{\mathcal{M}}, \quad \tilde{\mathcal{M}}_{2}=\left(\varepsilon_{w}^{\mathrm{pt}}\right)^{*} e^{i \mathbf{k}_{\mathrm{pt}} \cdot \mathbf{r}} \tilde{\mathcal{M}},
$$

where

$$
\tilde{\mathcal{M}}=\frac{\mu_{0} e^{2} \mathcal{N} V_{\mathrm{p}}}{2 m} \hbar \omega_{w} \frac{\mathbf{e}_{\mathrm{mol}} \cdot \mathbf{G}_{w}(\mathbf{r})}{\boldsymbol{\varepsilon}_{\mathrm{pt}} \cdot \mathbf{e}_{\mathrm{mol}}} .
$$

The energy is referenced to $\epsilon_{g}^{\mathrm{vib}}=0 . \mathbf{e}_{\mathrm{mol}}$ is a unit vector along the transition dipole moment of the molecule. All quantities that describe intermediate vibronic states cancel when deriving Eq. (21). Plasmonic enhancement in SERS is therefore independent of the molecule under study within the weak-coupling limit considered here.

The enhancement can be calculated with Eqs. (21), (22), and (23) in the vicinity of an arbitrary plasmonic nanostructure. The information about the LSP is contained in the plasmonic eigenvectors $\mathbf{q}_{w}(\mathbf{r})$. The eigenvectors need to be orthogonal,

$$
\int_{V_{\mathrm{p}}} d V^{\prime} \mathbf{q}_{w}\left(\mathbf{r}^{\prime}\right) \cdot \mathbf{q}_{w^{\prime}}\left(\mathbf{r}^{\prime}\right)=0
$$

for $w \neq w^{\prime}$ and must fulfill the normalization condition in Eq. (11). The eigenvectors $\mathbf{q}_{w}(\mathbf{r})$ may be obtained from the current distribution $\mathbf{j}(\mathbf{r})$ associated with the LSP mode $w$ [36]. Several approaches such as modal-expansion discretedipole approximation [42] and boundary-elements method eigenmode expansion [43] may be used to obtain eigenvectors. We use the quasistatic approximation and Mie theory to obtain analytic expressions for $\mathbf{q}_{w}(\mathbf{r})$ associated with the plasmon eigenmodes of a gold nanosphere and a nanosphere dimer. We stress that our HORa description of SERS is not limited to a specific theory for calculating the eigenvectors and matrix elements. The second quantization approach used here serves as an example to demonstrate the power and potential of our theory.

\section{SERS BY A GOLD NANOSPHERE}

\section{A. Adaption of model}

We apply our theory to a molecule that is coupled to the LSP of a gold nanosphere, which is the simplest model case for SERS. The LSP eigenvectors $\mathbf{q}_{w}(\mathbf{r})$ are obtained from the internal electric field in the gold nanosphere using Mie theory [Eq. (A3)]. Details on expressions and notation of Mie theory are provided in Appendix A. For light polarization along the $x$ axis the relevant plasmon eigenvectors are

$$
\mathbf{q}_{n}(\mathbf{r})=\xi E_{0} i^{n+1} \frac{2 n+1}{n(n+1)} d_{n}\left(\omega_{n}\right) \mathbf{N}_{e 1 n}^{(1)}(\mathbf{r}),
$$

with a normalization factor $\xi$. The index $n$ indicates the order of the LSP mode, i.e. dipole, quadrupole, etc., with degeneracy $(2 n+1)$ [43]. The Mie coefficient $\left|d_{n}(\omega)\right|^{2}$ becomes maximal at $\omega=\omega_{n}$, which we use to obtain the LSP energies $\hbar \omega_{n}$ (Appendix D). We dropped all terms related to the vector spherical harmonics $\mathbf{M}_{o 1 n}^{(1)}$ because they represent the excitation of magnetic resonances that are inherently weak in gold nanostructures [4]. The eigenvectors fulfill Eq. (24) because the vector spherical harmonics $\mathbf{N}_{e 1 n}^{(1)}(\mathbf{r})$ are orthogonal. We use $\sum_{w}=\sum_{n}(2 n+1)$ and rewrite Eq. (11) as

$$
V_{\mathrm{p}}=\sum_{n}(2 n+1) \int_{V_{\mathrm{p}}} d V \int_{V_{\mathrm{p}}} d V^{\prime} \mathbf{q}_{n}(\mathbf{r}) \cdot \mathbf{q}_{n}\left(\mathbf{r}^{\prime}\right)
$$

to calculate the normalization factor $\xi$. The integral vanishes for all $n \neq 1$ and we obtain

$$
\mathbf{q}_{n}(\mathbf{r})=\frac{i^{n+3} k_{n}^{3} \sqrt{V_{p}}(2 n+1) / n(n+1)}{\sqrt{48} \pi\left[k_{n} a \cos \left(k_{n} a\right)-\sin \left(k_{n} a\right)\right]} \frac{d_{n}\left(\omega_{n}\right)}{d_{1}\left(\omega_{1}\right)} \mathbf{N}_{e 1 n}^{(1)}(\mathbf{r}),
$$

where $a$ is the radius of the gold nanosphere. For the dipole plasmon mode ( $n=1$, in the following with index "pl") the expression simplifies to

$$
\mathbf{q}_{\mathrm{pl}}(\mathbf{r})=\frac{k_{\mathrm{pl}}^{3} \sqrt{3 V_{p}}}{8 \pi\left[k_{\mathrm{pl}} a \cos \left(k_{\mathrm{pl}} a\right)-\sin \left(k_{\mathrm{pl}} a\right)\right]} \mathbf{N}_{e 11}^{(1)}(\mathbf{r}) .
$$

As an alternative way to calculate the eigenvectors, we use the quasistatic point-dipole approximation. It is considered to be valid if the gold nanosphere is much smaller than the wavelength of the incident light. We obtain the LSP eigenvector from the induced dipole moment in the sphere center

$$
\mathbf{p}_{\mathrm{pl}}(\mathbf{r}, \omega)=\alpha(\omega) E_{0} \boldsymbol{\varepsilon}_{\mathrm{pt}} \delta(\mathbf{r}),
$$

where $\alpha$ is the polarizability of the gold nanosphere; see Eq. (C3). Normalization leads to the simple expression

$$
\mathbf{q}_{\mathrm{pl}}(\mathbf{r})=\sqrt{\frac{V_{\mathrm{p}}}{3}} \boldsymbol{\varepsilon}_{\mathrm{pt}} \delta(\mathbf{r})
$$

With the explicit expression for the plasmon eigenvector, we have now all ingredients at hand to calculate the plasmonic SERS enhancement by a single nanosphere using Eq. (21). In the following, we restrict our calculations to the dipole plasmon mode, which provides the strongest enhancement. For a molecule with its transition dipole moment oriented along the polarization of the incoming light we find the coupling factors

$$
\tilde{\mathcal{M}}_{1}(\mathbf{r})=-\frac{\mu_{0} e^{2} \mathcal{N} V_{\mathrm{p}} \hbar \omega_{\mathrm{pl}} k_{\mathrm{pl}}}{24 \pi m} \beta(\mathbf{r})
$$


(a)

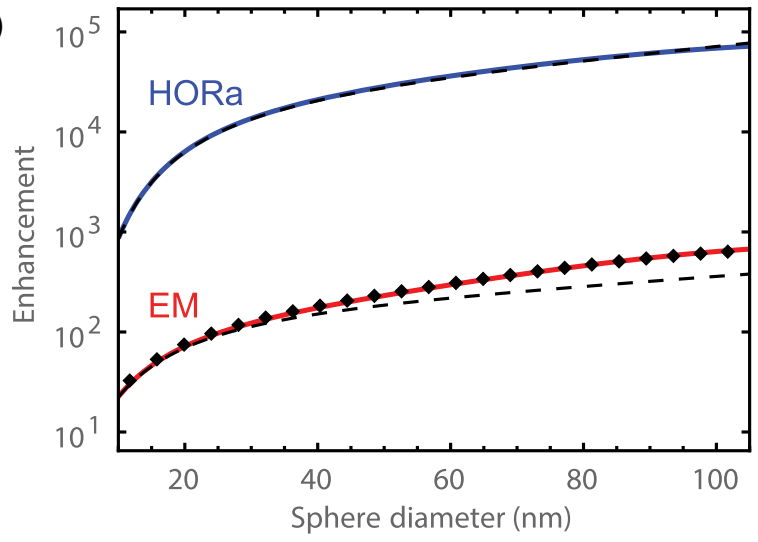

(b)

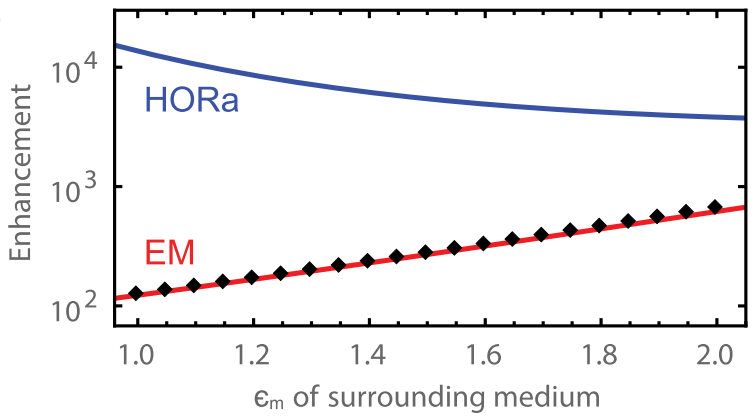

FIG. 3. Plasmonic enhancement from SERS as higher-order Raman scattering (HORa) and EM enhancement (EM) for a molecule positioned $2 \mathrm{~nm}$ beside a gold nanosphere. (a) Maximum enhancement as a function of sphere diameter $\left(\epsilon_{\mathrm{m}}=1\right)$. Solid lines correspond to enhancement from calculations based on Mie theory and dashed lines correspond to quasistatic approximation. Diamonds mark the enhancement obtained by EM-DR. (b) Maximum enhancement for 30-nm gold nanosphere as a function of dielectric constant $\epsilon_{\mathrm{m}}$ of the surrounding medium. (Parameters: Raman shift of $1000 \mathrm{~cm}^{-1}$, $\mathbf{e}_{\text {mol }} \| \boldsymbol{\varepsilon}_{\mathrm{pt}}, \mathcal{N}=5.9 \times 10^{22} \mathrm{~cm}^{-3}, \omega_{\mathrm{pl}}$, and $\gamma_{\mathrm{pl}}$ from fit of $\left|d_{1}(\omega)\right|^{2}$; see Appendix D.)

and $\tilde{\mathcal{M}}_{2}=-\tilde{\mathcal{M}}_{1} e^{2 i k_{\mathrm{p} 1} r \cos \vartheta} . \beta$ is a dimensionless factor that describes the position dependence of the coupling. Using the plasmon eigenvector within the quasistatic approximation, Eq. (30), we find

$$
\beta(r, \vartheta=\pi / 2, \varphi=0)=2 e^{i k_{\mathrm{p} 1} r} \frac{1-i k_{\mathrm{pl}} r}{k_{\mathrm{pl}}^{3} r^{3}},
$$

for the enhancement along the $x$ axis $\left(\boldsymbol{\varepsilon}_{\mathrm{pt}}=\mathbf{e}_{x}, \mathbf{k}_{\mathrm{pt}}=k_{\mathrm{pl}} \mathbf{e}_{z}\right.$, sphere center at origin). Explicit expressions for $\beta$ derived from the LSP eigenvector within Mie theory, Eq. (28), are given in Appendix B.

\section{B. Calculation of SERS enhancement}

We calculate the SERS enhancement for a molecule coupled to the LSP dipole mode of a gold nanosphere as a function of sphere size, dielectric environment, and position of the molecule (Figs. 3 and 4). Energy $\hbar \omega_{\mathrm{pl}}$ and spectral width $2 \gamma_{\mathrm{pl}}$ of the LSP mode are obtained from Mie theory. It predicts values that nicely resemble experimental data [44] (see Appendix D). We compare the calculated enhancement treating SERS as a higher-order Raman process within perturbation theory (a)
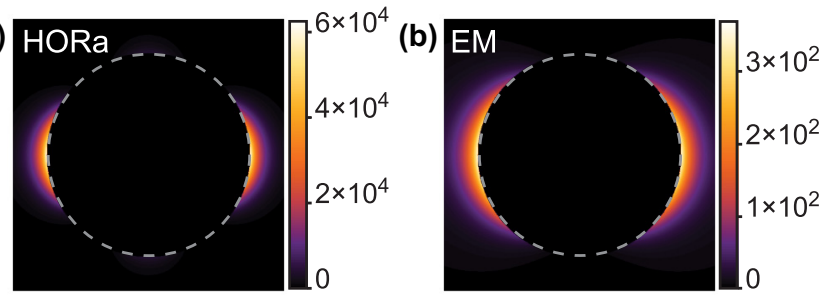

(c)
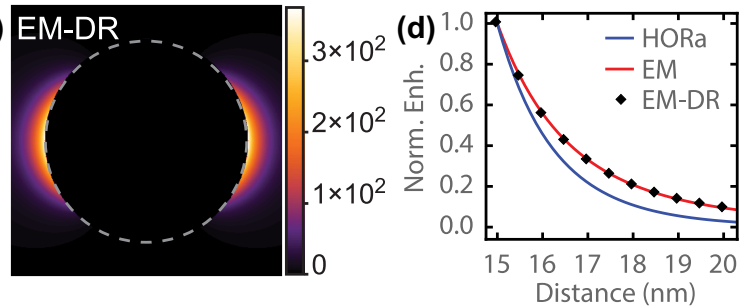

FIG. 4. Geometry of a SERS hot spot. Maximum enhancement is calculated as a function of molecule position close to a 30-nm gold nanosphere from (a) SERS within higher-order Raman scattering (HORa), (b) EM model, and (c) EM-DR model. (d) Maximum enhancement along the $x$ axis as a function of distance to the sphere center. Enhancement is normalized to the enhancement at the sphere surface $(x=15 \mathrm{~nm})$. (Parameters: Raman shift of $1000 \mathrm{~cm}^{-1}$, $\mathbf{e}_{\mathrm{mol}} \| \boldsymbol{\varepsilon}_{\mathrm{pt}}, \epsilon_{\mathrm{m}}=1, \mathcal{N}=5.9 \times 10^{22} \mathrm{~cm}^{-3}, \omega_{\mathrm{pl}}$, and $\gamma_{\mathrm{pl}}$ from fit of $\left|d_{1}(\omega)\right|^{2}$; see Appendix D.)

to that obtained from the theory of EM enhancement. EM enhancement is calculated as the product of the local electricfield intensities at $\omega_{\mathrm{L}}$ and $\omega_{\mathrm{S}}$; see Eq. (C1) in Appendix C. We further calculate the EM enhancement with the EM model including dipole radiation effects (EM-DR) [8,9].

Our HORa description of SERS [blue line in Fig. 3(a), HORa, Eq. (21)] predicts $10^{3}$ enhancement of the Raman cross section for a molecule positioned $2 \mathrm{~nm}$ away from an $\mathrm{Au}$ sphere with a diameter of $10 \mathrm{~nm}$. The enhancement increases to $\sim 10^{5}$ for a sphere diameter of $100 \mathrm{~nm}$. The EM enhancement is two orders of magnitude weaker [red line in Fig. 3(a), EM]. EM and EM-DR models lead to almost identical enhancement (diamond plot markers). Our approach thus predicts a strikingly stronger plasmonic enhancement in SERS than the theory of EM enhancement. The question arises how the theories compare to experiment. It is challenging to compare the SERS enhancement from experiments with that predicted by theory because of the uncertainties involved. Nevertheless, in a recent experiment colocalized atomic force and SERS microscopic imaging were used to characterize individual gold nanoparticles coated with molecules [13]. The measured SERS enhancement for gold nanorods $\left(10^{6}-10^{7}\right)$ was two to three orders of magnitude stronger than the calculated EM enhancement. The discrepancy was attributed to chemical enhancement, which could not be quantified. From our HORa calculations we conclude plasmonic enhancement accounts for most of the SERS intensity; it was underestimated by the EM-enhancement theory.

Another interesting point in Fig. 3(a) is the comparison between Mie theory (solid lines) and the quasistatic approximation (dashed). The quasistatic approximation for the HORa calculations coincides surprisingly well with Mie theory. This arises because the normalization factor $\xi$ in Eq. (25) decreases 
with sphere diameter within Mie theory, which is not the case within the quasistatic approximation. The much simpler equations based on the quasistatic approximation can therefore be used to accurately calculate the enhancement from our approach around a nanosphere of arbitrary size. The quasistatic calculations with the theory of EM enhancement, in contrast, underestimate the enhancement for nanospheres larger than $40 \mathrm{~nm}$.

The HORa description of SERS predicts the opposite dependence of the plasmonic enhancement on the dielectric constant $\epsilon_{\mathrm{m}}$ of the surrounding medium compared to the EM-enhancement model [Fig. 3(b)]. An increase in refractive index $\sqrt{\epsilon_{\mathrm{m}}}$ leads to a decrease in enhancement for HORa calculations, whereas the EM enhancement increases. The difference between the models is therefore less pronounced for the typical environments encountered in SERS experiments, such as water $\left(\epsilon_{\mathrm{m}}=1.77\right)$ or a $\mathrm{SiO}_{2}$ substrate $\left(\epsilon_{\mathrm{m}, \text { eff }}<\right.$ 1.5) [45]. The dependence of the SERS enhancement on dielectric environment may thus serve as an experimental prove of our theory.

We calculate the plasmonic enhancement as a function of molecule position close to a 30-nm gold nanosphere (Fig. 4). The transition dipole moment of the molecule is parallel to the $x$ axis (i.e., $\mathbf{e}_{\text {mol }} \| \boldsymbol{\varepsilon}_{\mathrm{pt}}$ ). Our approach [Fig. 4(a)] predicts in this case a spatially more confined hot spot than EM [Fig. 4(b)] and EM-DR [Fig. 4(c)]. This has two origins. First, the polarization of the plasmonic near field is included within our approach. Coupling is strongest for $\mathbf{E}_{\mathrm{pl}} \| \mathbf{e}_{\mathrm{mol}}$ but vanishes for $\mathbf{E}_{\mathrm{pl}} \perp \mathbf{e}_{\mathrm{mol}}$. Moving away from the $x$ axis, $\mathbf{E}_{\mathrm{pl}}$ is no longer parallel to $\mathbf{e}_{\mathrm{mol}}$ (see field lines in Fig. 2, which shows a similar geometry for a nanoparticle dimer) leading to a more strongly confined hot spot than EM enhancement. The near-field polarization is also included in the EM-DR calculation, resulting likewise in a more localized hot spot than EM enhancement. The difference between HORa and EM-DR is explained by the stronger total enhancement predicted by our description of SERS $\left(\sim 10^{4}\right.$ for HORa and $\sim 10^{2}$ for EM-DR). Strong enhancement leads to a dominance of the scattering process where incoming and scattered light couple to the plasmon [Fig. 1(b)] with an $\sim 1 / r^{12}$ dependence along the $x$ axis, as can be estimated from Eqs. (21), (31), and (32); see blue line in Fig. 4(d). In the enhancement regime predicted from the EM-DR model, scattering processes in which either the incoming light or the scattered light couple to the plasmon become more important [Figs. 1(d) and 1(e)]. The enhancement follows an $\sim 1 / r^{9}$ dependence [see diamonds in Fig. 4(d)]. This accounts for the more extended hot spot of EM-DR in Fig. 4(c). Overall, there is good agreement between the hot-spot geometries predicted from our theory and EM or EM-DR theory. Both theories lead to the same design criteria for SERS substrates, which is important for the modeling of nanostructures.

\section{ENHANCEMENT IN GOLD NANODIMER}

\section{A. Adaption of model}

A single nanosphere as discussed in Sec. III is an interesting model system for plasmonic enhancement and frequently studied theoretically. However, its enhancement is usually too weak for SERS experiments. The general consensus within the community is that SERS arises from dimers or clusters of nanoparticles $[11,47]$. We therefore apply our theory to a molecule that is placed in the plasmonic hot spot of a gold nanoparticle dimer. We restrict the calculations to the lowest-order dipole mode $w=1$ (here with index "pl"), with frequency $\omega_{\mathrm{pl}}$ and light polarization along the dimer axis. The plasmon eigenvector $\mathbf{q}_{\mathrm{pl}}(\mathbf{r})$ is obtained from the induced dipole moment $\mathbf{p}_{\mathrm{pl}}(\mathbf{r})$, Eq. (C7), in the quasistatic point-dipole approximation. By normalizing with respect to the three dipole modes of the nanodimer (one $\|$ and two $\perp$ to the dimer axis), we find

$$
\mathbf{q}_{\mathrm{pl}}(\mathbf{r})=\xi(d) \sqrt{V_{\mathrm{p}}} \frac{\mathbf{d}}{d}[\delta(\mathbf{r}-\mathbf{d} / 2)+\delta(\mathbf{r}+\mathbf{d} / 2)],
$$

with a normalization factor

$$
\xi(d)=\frac{\alpha_{\|}^{\text {ind }}\left(\omega_{\mathrm{pl}, \|}, d\right)}{2 \sqrt{\left[\alpha_{\|}^{\text {ind }}\left(\omega_{\mathrm{pl}, \|}, d\right)\right]^{2}+2\left[\alpha_{\perp}^{\text {ind }}\left(\omega_{\mathrm{pl}, \perp}, d\right)\right]^{2}}},
$$

where d connects the two sphere centers and $d=|\mathbf{d}|$. The polarizabilities $\alpha_{\|}^{\text {ind }}$ and $\alpha_{\perp}^{\text {ind }}$ of the gold nanodimer are derived in Appendix C. Note that the point-dipole approximation is valid only for $d>3 a$. A formalism that goes beyond this approximation must be based on generalized Mie theory and will be the subject of future work.

Using Eq. (33), we find the coupling factors

$$
\tilde{\mathcal{M}}_{1}(d)=-\frac{4 \mu_{0} e^{2} \mathcal{N} V_{\mathrm{p}} \hbar \omega_{\mathrm{pl}} k_{\mathrm{pl}}}{\pi m} \beta(d)
$$

and $\tilde{\mathcal{M}}_{2}=-\left[\xi^{*}(d) / \xi(d)\right] \tilde{\mathcal{M}}_{1}$, with

$$
\beta(d)=\xi^{2}(d) e^{i k_{\mathrm{pl}} d / 2} \frac{2-i k_{\mathrm{pl}} d}{k_{\mathrm{pl}}^{3} d^{3}}
$$

for the plasmonic enhancement in the center of the nanodimer.

\section{B. Calculation of SERS enhancement}

We calculate the SERS enhancement for a molecule in a gold nanodimer with $30 \mathrm{~nm}$ sphere diameters as a function of gap size and dielectric constant $\epsilon_{\mathrm{m}}$ of the surrounding medium [Fig. 5(a)]. $\omega_{\mathrm{pl}}$ and $\gamma_{\mathrm{pl}}$ are obtained from a fit of the polarizability $\left|\alpha_{\|}^{\text {ind }}(\omega, d)\right|^{2}$; see Appendix D . For a gap of $15 \mathrm{~nm}$ and $\epsilon_{\mathrm{m}}=1$, our HORa description of SERS predicts an enhancement $\left(3.8 \times 10^{4}\right)$ that is $\sim 350$ times larger than the EM enhancement $\left(1.1 \times 10^{2}\right)$. The ratio gets even larger for smaller gap sizes and increases to three orders of magnitude for a gap of $5 \mathrm{~nm}$ [48]. This increase can be attributed to the quasiparticle description of the LSP. The normalization factor $\xi(d)$ increases with the coupling between the two nanospheres, i.e., with decreasing gap size. The dipole mode along the dimer axis dominantes over the modes perpendicular to the dimer axis and the degeneracy between the three dipole modes is lifted. In Fig. 5(a), we also plot the difference in enhancement predicted by our approach and EM enhancement for water $\left(\epsilon_{\mathrm{m}}=1.77\right)$ and $\mathrm{a} \mathrm{SiO}_{2}$ substrate with $\epsilon_{\mathrm{m}, \text { eff }}=1.4$. The difference in enhancement decreases with increasing refractive index $\sqrt{\epsilon_{\mathrm{m}}}$ of the environment, similar to the case of a single sphere. The excitation-energy dependence of the plasmonic enhancement shows a symmetric Lorentzian resonance profile from an overlapping incoming and outgoing resonance [blue line in 
(a)

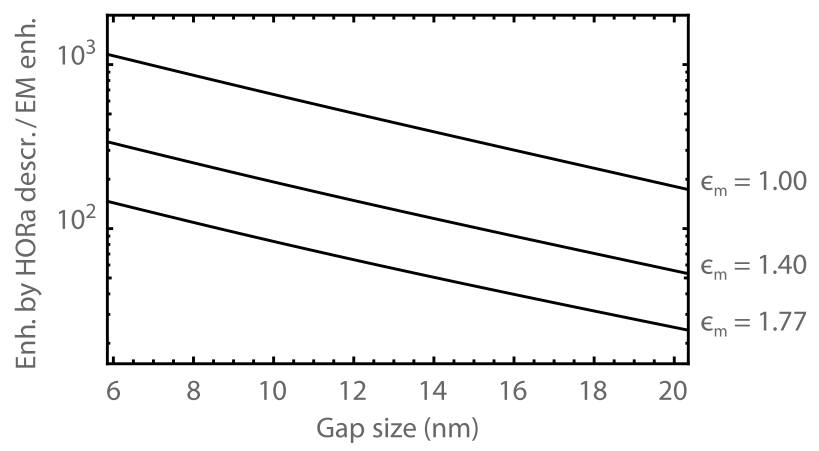

(b)

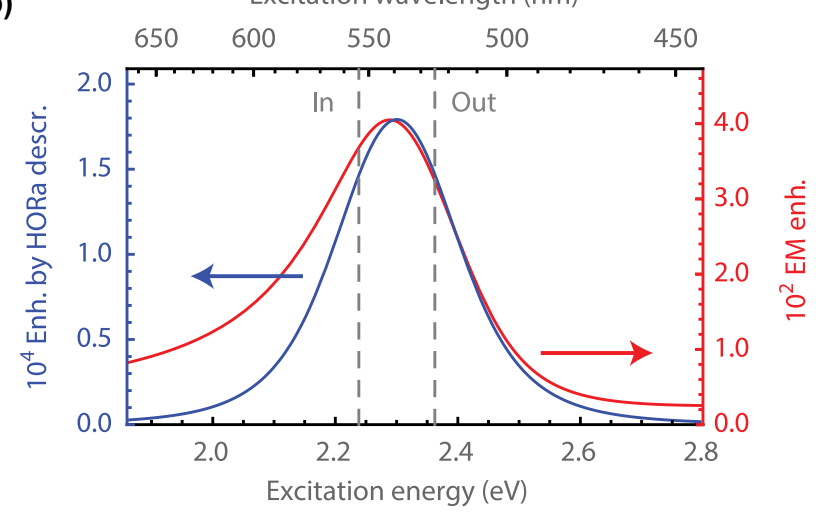

FIG. 5. Plasmonic enhancement from our description of SERS as higher-order Raman scattering (HORa) and EM-enhancement model in the hot spot of a gold nanodimer. (a) Maximum enhancement from HORa description divided by maximum EM enhancement as a function of gap size for three typical values of $\epsilon_{\mathrm{m}}$. (b) Comparison of excitation-energy dependence of enhancement from HORa description and EM-enhancement model for a gap of $15 \mathrm{~nm}$ and water as the surrounding medium $\left(\epsilon_{\mathrm{m}}=1.77\right)$. Energies of incoming and scattered light are indicated by dashed lines. (Parameters: $30 \mathrm{~nm}$ sphere diameters, Raman shift of $1000 \mathrm{~cm}^{-1}$, $\mathcal{N}=5.9 \times 10^{22} \mathrm{~cm}^{-3}, \omega_{\mathrm{pl}}$, and $\gamma_{\mathrm{pl}}$ obtained from fit of $\left|\alpha_{\|}^{\text {ind }}(\omega, d)\right|^{2} ;$ see Appendix D.)

Fig. $5(\mathrm{~b}) ; \gamma_{\mathrm{pl}} \approx 0.15 \mathrm{eV}$ ]. The resonance is more pronounced than within the EM-enhancement model [red line in Fig. 5(b)].

In recent experiments with gold nanoparticle dimers and trimers a combination of Raman spectroscopy and electron microscopy was used to obtain a structure-activity relationship for SERS $[10-12,49]$. In all experiments the measured SERS enhancement was considerably higher than the calculated EM enhancement. Crozier and co-workers measured two to four orders of magnitude difference to the EM enhancement for lithographically fabricated gold nanodimers on $\mathrm{SiO}_{2}$ and $\mathrm{Si}_{3} \mathrm{~N}_{4}$ substrates as plasmonic nanostructure [11,12]. Van Duyne and co-workers used molecule-coated dimers and trimers of chemically synthesized gold nanoparticles encapsulated in a $\mathrm{SiO}_{2}$ shell $[10,49]$. The EM-enhancement theory underestimated the measured values by factors between 50 and 100. The discrepancy was argued to arise from chemical enhancement. Chemical enhancement is expected to give rise to both enhancement and weakening of the Raman response depending on the details of the system under study $[4,16]$. In view of this, a consistent increase in all experiments appears quite surprising. The description of SERS as a higher-order Raman process excellently explains the experimental findings. Experiments in which chemical enhancement is excluded through the choice of nanoplasmonic system and Raman probe are highly desirable to compare plasmonic enhancement in theory and experiment.

\section{PRIOR QUANTUM-MECHANICAL SERS THEORIES}

A number of quantum-mechanical approaches to SERS have been proposed in the last decade. In this section, we want to elucidate differences and similarities to our approach. It is not our intent to provide an exhaustive summary of all previous theoretical work; our discussion focuses on selected studies that we found representative for other approaches as well. The main idea of our microscopic theory is the description of SERS as a higher-order Raman process. We therefore focus our discussion on the treatment of the Raman process in the previous quantum-mechanical approaches.

Jensen and co-workers used an atomistic electrodynamics model for the metal nanoparticle and described the molecule with time-dependent density functional theory $[17,18]$. This hybrid quantum-mechanics-classical-electrodynamics approach was demonstrated to be particularly suitable for describing the metal-molecule interaction at atomic length scales. The description of the Raman effect was based on the Kramers-Heisenberg-Dirac formalism, which is secondorder perturbation theory [50]. It thereby differed from our description of surface-enhanced Raman scattering as thirdand fourth-order scattering processes. In contrast to our work, calculated enhancement factors were identical with EM enhancement for metal-molecule distances larger than $6 \AA$, whereas deviations of less than an order of magnitude were found for smaller distances [18].

A microscopic theory of SERS based on a many-body Green's function approach was proposed by Masiello and Schatz $[19,20]$. The molecule was treated with first-principles quantum mechanics, whereas the LSP was described with classical electrodynamics. The repeated interaction of a molecular dipole with its image in a nearby metallic nanoparticle was demonstrated to become particularly important in the strong-coupling regime [21]. The metal-molecule interaction was treated up to infinite order in perturbation theory. This is not to be confused with the description of the Raman effect, which was contrary to our approach described as first-order Raman scattering with second-order perturbation theory [19].

A quasiclassical description of SERS based on the Purcell effect was proposed by Gu et al. [25]. While the enhancement of the incoming light was calculated in the same way as in the EM-enhancement theory, the enhancement of the scattered light was calculated with the Purcell effect using Fermi's golden rule. The approach thereby used the same implicit description of the Raman effect as the EM-enhancement theory. Predictions from this model agreed with the EMenhancement theory.

Kelley proposed a fully quantum-mechanical treatment of SERS based on the density matrix formalism [22]. SERS and Raman scattering in the absence of a plasmonic nanostructure were both described with a perturbative expansion of the density matrix up to fourth order. Molecules and plasmonic 
nanoparticles were assumed to interact through Förster-type energy transfer, which lead to mixed states. The SERS enhancement was strongly affected by the electronic resonances of the molecule. The enhancement was found to be the largest for a molecular resonance far away from the plasmonic resonance of the nanoparticle. This theory goes, similar to our approach, beyond the external plasmonic enhancement description of SERS and treats molecule and nanoparticle transitions on equal footing. It is, however, limited to the strong-coupling regime, which is uncommon in SERS.

\section{CONCLUSION}

We propose to model SERS as a higher-order Raman process. The plasmonic excitation is treated as an integral part of the Raman scattering process. Our approach modifies the widely employed microscopic theory of Raman scattering to correctly describe SERS. The interaction Hamiltonians involving plasmonic excitations were derived using second quantization of the LSP. This perturbative approach enabled us to obtain analytic expressions for all coupling matrix elements with no further need for numerical implementation. Chemical interactions between the metal and molecule wave functions can be included within perturbation theory with Herzberg-Teller terms, as demonstrated by Lombardi and Birke [15,34]. Furthermore, our approach can be also applied to SERS in solid-state materials, such as graphene [51,52]. In this case all Raman amplitudes must be calculated one order higher in perturbation theory because of decoupled vibrational and electronic states.

We demonstrated the power of understanding SERS as higher-order Raman scattering by calculating the plasmonic enhancement of the Raman scattering cross section for a molecule coupled to the LSP of a gold nanosphere and a nanosphere dimer. Our theory leads to the same design criteria for plasmonic nanostructures as the EM and EM-DRenhancement theory and accounts for the polarization of the plasmonic near field. The predicted enhancement close to the gold nanosphere was up to two orders of magnitude larger than expected from electromagnetic enhancement, a difference that increased to up to three orders of magnitude in the hot spot of a nanodimer. The prediction of stronger plasmonic enhancement is attributed to the proper description of SERS as a higher-order Raman effect. It explains the enhancement factors extracted in recent SERS experiments [10-13,49] and furthermore highlights the dominance of plasmonic enhancement in SERS. Our theory is an important step towards a unified quantummechanical description of SERS.

\section{ACKNOWLEDGMENTS}

We acknowledge the NanoScale Focus Area and Deutsche Forschungsgemeinschaft Grant No. SFB658. N.M. thanks Deutsche Telekom Stiftung.

\section{APPENDIX A: MIE THEORY}

In this Appendix we provide all relevant expressions of Mie theory that were used in this work. We use the notation by Bohren and Huffman [53]. Consider a plane wave with amplitude $E_{0}$, polarization along the $x$ axis, and propagation along the $z$ axis as incident light field

$$
\mathbf{E}_{\mathrm{inc}}=E_{0} e^{i k r \cos \vartheta} \hat{\mathbf{e}}_{x} .
$$

This expression can be expanded in vector spherical harmonics (VSH),

$$
\mathbf{E}_{\mathrm{inc}}=E_{0} \sum_{n=1}^{\infty} i^{n} \frac{2 n+1}{n(n+1)}\left(i \mathbf{N}_{e 1 n}^{(1)}-\mathbf{M}_{o 1 n}^{(1)}\right) .
$$

The induced electric field inside the nanosphere is

$$
\mathbf{E}_{\mathrm{int}}=E_{0} \sum_{n=1}^{\infty} i^{n} \frac{2 n+1}{n(n+1)}\left(i d_{n} \mathbf{N}_{e 1 n}^{(1)}-c_{n} \mathbf{M}_{o 1 n}^{(1)}\right),
$$

and the scattered electric field around the nanoparticle is

$$
\mathbf{E}_{\mathrm{sca}}=E_{0} \sum_{n=1}^{\infty} i^{n} \frac{2 n+1}{n(n+1)}\left(b_{n} \mathbf{M}_{o 1 n}^{(3)}-i a_{n} \mathbf{N}_{e 1 n}^{(3)}\right) .
$$

The VSH are defined as

$$
\mathbf{M}_{e m n}=\nabla \times\left(\mathbf{r} \Psi_{e m n}\right), \quad \mathbf{M}_{\text {omn }}=\nabla \times\left(\mathbf{r} \Psi_{o m n}\right),
$$

and

$$
\mathbf{N}_{e m n}=\frac{\nabla \times \mathbf{M}_{e m n}}{k}, \quad \mathbf{N}_{o m n}=\frac{\nabla \times \mathbf{M}_{o m n}}{k},
$$

where

$$
\begin{aligned}
& \Psi_{e m n}=\cos (m \varphi) P_{n}^{m}(\cos \vartheta) z_{n}(k r), \\
& \Psi_{o m n}=\sin (m \varphi) P_{n}^{m}(\cos \vartheta) z_{n}(k r) .
\end{aligned}
$$

Here $e$ stands for even and $o$ for odd; $P_{n}^{m}$ is the associated Legendre polynomial. The superscript (1) specifies $z_{n}(k r)=j_{n}(k r)$ and (3) specifies $z_{n}(k r)=h_{n}^{(1)}(k r)$, where $j_{n}$ is the spherical Bessel function and $h_{n}^{(1)}$ the spherical Hankel function of the first kind. The VSH satisfy orthogonality in a sense that

$$
\int_{0}^{\pi} d \vartheta \sin \vartheta \int_{0}^{2 \pi} d \varphi \mathbf{N}_{e m n} \cdot \mathbf{N}_{e m^{\prime} n^{\prime}}=0,
$$

if $n \neq n^{\prime}$ or $m \neq m^{\prime}$. Similarly, $\left(\mathbf{M}_{e m n}, \mathbf{M}_{e m^{\prime} n^{\prime}}\right),\left(\mathbf{N}_{o m n}, \mathbf{N}_{o m^{\prime} n^{\prime}}\right)$, and $\left(\mathbf{M}_{o m}, \mathbf{M}_{o m^{\prime} n^{\prime}}\right)$ are orthogonal sets for $n \neq n^{\prime}$ or $m \neq m^{\prime}$. Additionally, VSH of different kinds form orthogonal sets, i.e., $\left(\mathbf{N}_{e m n}, \mathbf{M}_{e m^{\prime} n^{\prime}}\right),\left(\mathbf{N}_{e m n}, \mathbf{N}_{o m^{\prime} n^{\prime}}\right)$, etc., for all $n, n^{\prime}, m, m^{\prime}$. The optical response of the nanosphere is described by the Mie coefficients

$$
\begin{aligned}
& a_{n}= \frac{s \psi_{n}(s \rho) \psi_{n}^{\prime}(\rho)-\psi_{n}(\rho) \psi_{n}^{\prime}(s \rho)}{s \psi_{n}(s \rho) \xi_{n}^{\prime}(\rho)-\xi_{n}(\rho) \psi_{n}^{\prime}(s \rho)}, \\
& b_{n}=\frac{\psi_{n}(s \rho) \psi_{n}^{\prime}(\rho)-s \psi_{n}(\rho) \psi_{n}^{\prime}(s \rho)}{\psi_{n}(s \rho) \xi_{n}^{\prime}(\rho)-s \xi_{n}(\rho) \psi_{n}^{\prime}(s \rho)}, \\
& c_{n}=\frac{s \psi_{n}(\rho) \xi_{n}^{\prime}(\rho)-s \xi_{n}(\rho) \psi_{n}^{\prime}(\rho)}{\psi_{n}(s \rho) \xi_{n}^{\prime}(\rho)-s \xi_{n}(\rho) \psi_{n}^{\prime}(s \rho)}, \\
& d_{n}=\frac{s \psi_{n}(\rho) \xi_{n}^{\prime}(\rho)-s \xi_{n}(\rho) \psi_{n}^{\prime}(\rho)}{s \psi_{n}(s \rho) \xi_{n}^{\prime}(\rho)-\xi_{n}(\rho) \psi_{n}^{\prime}(s \rho)},
\end{aligned}
$$


with

$$
\psi_{n}(\rho)=\rho j_{n}(\rho), \quad \xi_{n}(\rho)=\rho h^{(1)}(\rho)
$$

and their derivatives denoted by " " ". We used the substitutions $\rho=k a$ and $s=\sqrt{\epsilon_{\mathrm{Au}} / \epsilon_{\mathrm{m}}}$, with the dielectric function of gold $\epsilon_{\mathrm{Au}}$ and the sphere radius $a$.

\section{APPENDIX B: COUPLING FACTORS FOR SERS BESIDE A SINGLE NANOSPHERE}

The coupling factors $\tilde{\mathcal{M}}_{1}$ and $\tilde{\mathcal{M}}_{2}$ in Eq. (22) contain the integrals $\varepsilon_{\mathrm{pl}}^{\mathrm{pt}}$ and $\mathbf{G}_{\mathrm{pl}}(\mathbf{r})$. We calculate $\varepsilon_{\mathrm{pl}}^{\mathrm{pt}}$ from Eq. (18) by using the expansion of a plane wave into VSH in Eq. (A2),

$$
\varepsilon_{\mathrm{pl}}^{\mathrm{pt}}=-\sum_{n=1}^{\infty} i^{n} \frac{2 n+1}{n(n+1)} \frac{1}{\sqrt{V_{\mathrm{p}}}} \int_{V_{\mathrm{p}}} d V^{\prime}\left[\mathbf{M}_{o 1 n}^{(1)}\left(\mathbf{r}^{\prime}\right)-i \mathbf{N}_{e 1 n}^{(1)}\left(\mathbf{r}^{\prime}\right)\right] \cdot \mathbf{q}_{\mathrm{pl}}\left(\mathbf{r}^{\prime}\right) .
$$

The integral vanishes except for $n=1$ because of $\mathbf{q}_{\mathrm{pl}} \propto \mathbf{N}_{e 11}^{(1)}$ and the orthogonality of the VSH. With Eq. (28), we obtain the analytic expression

$$
\varepsilon_{\mathrm{pl}}^{\mathrm{pt}}(\rho)=\frac{\sqrt{3}}{4} \frac{1+\rho^{2}-\rho^{4}+\left(-1+\rho^{2}\right) \cos (2 \rho)+\rho\left(-2+\rho^{2} / 2\right) \sin (2 \rho)}{\rho^{3}(\rho \cos \rho-\sin \rho)},
$$

where $\rho=k_{\mathrm{pl}} a$. To calculate $\mathbf{G}_{\mathrm{pl}}(\mathbf{r})$, we first express the dyadic Green's function in terms of the Helmholtz Green's function,

$$
G_{0}\left(\mathbf{r}, \mathbf{r}^{\prime}\right)=\frac{e^{i k\left|\mathbf{r}-\mathbf{r}^{\prime}\right|}}{4 \pi\left|\mathbf{r}-\mathbf{r}^{\prime}\right|}
$$

as

$$
\stackrel{\leftrightarrow}{\mathbf{G}}\left(\mathbf{r}, \mathbf{r}^{\prime}\right)=\left(\stackrel{\leftrightarrow}{\mathbf{I}}+\frac{1}{k^{2}} \nabla_{\mathbf{r}} \nabla_{\mathbf{r}}\right) G_{0}\left(\mathbf{r}, \mathbf{r}^{\prime}\right)
$$

with the abbreviation $\nabla G_{0}=\nabla \cdot\left(G_{0} \stackrel{\leftrightarrow}{\mathbf{I}}\right)$ [41]. We use an expansion of the Helmholtz Green's function,

$$
G_{0}\left(\mathbf{r}, \mathbf{r}^{\prime}\right)=i k \sum_{l=0}^{\infty} j_{l}\left(k r^{\prime}\right) h_{l}^{(1)}(k r) \sum_{m=-l}^{l} Y_{l m}^{*}\left(\vartheta^{\prime}, \varphi^{\prime}\right) Y_{l m}(\vartheta, \varphi)
$$

in spherical harmonics $Y_{l m}$ [54]. We first calculate with Eq. (28) for the dipole plasmon mode,

$$
\begin{aligned}
\int_{V_{\mathrm{p}}} d V^{\prime} G_{0}\left(\mathbf{r}, \mathbf{r}^{\prime}\right) \mathbf{q}_{\mathrm{pl}}\left(\mathbf{r}^{\prime}\right)= & \frac{i k_{\mathrm{pl}}^{4} \sqrt{3 V_{p}}}{8 \pi\left[k_{\mathrm{pl}} a \cos \left(k_{\mathrm{pl}} a\right)-\sin \left(k_{\mathrm{pl}} a\right)\right]} \sum_{l=0}^{\infty} h_{l}^{(1)}\left(k_{\mathrm{pl}} r\right) \sum_{m=-l}^{l} Y_{l m}(\vartheta, \varphi) \\
& \times \int_{0}^{a} d r^{\prime} r^{\prime 2} \int_{0}^{\pi} d \vartheta^{\prime} \sin \vartheta^{\prime} \int_{0}^{2 \pi} d \varphi^{\prime} j_{l}\left(k_{\mathrm{pl}} r^{\prime}\right) Y_{l m}^{*}\left(\vartheta^{\prime}, \varphi^{\prime}\right) \mathbf{N}_{e 11}^{(1)}\left(k_{\mathrm{pl}} r^{\prime}, \vartheta^{\prime}, \varphi^{\prime}\right) .
\end{aligned}
$$

The integral vanishes except for $l=0$ and $l=2$ and we obtain

$$
\begin{aligned}
\int_{V_{\mathrm{p}}} d V^{\prime} G_{0}\left(\mathbf{r}, \mathbf{r}^{\prime}\right) \mathbf{q}_{\mathrm{pl}}\left(\mathbf{r}^{\prime}\right)= & \frac{\sqrt{3 V_{\mathrm{p}}}}{8 \pi \rho^{3}(\rho \cos \rho-\sin \rho)} \frac{e^{i k_{\mathrm{pl}} r}}{k_{\mathrm{pl}}^{2} r^{3}}\left\{k_{\mathrm{pl}}^{2} r^{2} \rho^{3} \frac{-2 \rho+\sin (2 \rho)}{6} \mathbf{e}_{x}+\left[-6-6 \rho^{2}+2 \rho^{4}+\left(6-6 \rho^{2}\right) \cos (2 \rho)\right.\right. \\
& \left.\left.-\rho\left(-12+\rho^{2}\right) \sin (2 \rho)\right]\left(-3+3 i k_{\mathrm{pl}} r+k_{\mathrm{pl}}^{2} r^{2}\right)\left(2 \sin \vartheta \cos \varphi \mathbf{e}_{r}-\cos \vartheta \cos \varphi \mathbf{e}_{\vartheta}+\sin \varphi \mathbf{e}_{\varphi}\right) / 12\right\} .
\end{aligned}
$$

As a last step we calculate $\mathbf{G}_{\mathrm{pl}}(\mathbf{r})$ in Eq. (16) using the vector Laplacian in spherical coordinates,

$$
\begin{aligned}
\mathbf{G}_{\mathrm{pl}}(r, \vartheta, \varphi, \rho)= & \frac{\sqrt{3}}{8 \pi \rho^{3}(\rho \cos \rho-\sin \rho)} \frac{e^{i k_{\mathrm{pl}} r}}{k_{\mathrm{pl}}^{2} r^{3}}\left\{k_{\mathrm{pl}}^{2} r^{2} \rho^{3} \frac{-2 \rho+\sin (2 \rho)}{6} \mathbf{e}_{x}+\left[-6-6 \rho^{2}+2 \rho^{4}+\left(6-6 \rho^{2}\right) \cos (2 \rho)\right.\right. \\
& \left.-\rho\left(-12+\rho^{2}\right) \sin (2 \rho)\right]\left(-3+3 i k_{\mathrm{pl}} r+k_{\mathrm{pl}}^{2} r^{2}\right)\left(2 \sin \vartheta \cos \varphi \mathbf{e}_{r}-\cos \vartheta \cos \varphi \mathbf{e}_{\vartheta}+\sin \varphi \mathbf{e}_{\varphi}\right) / 12 \\
& \left.-2(\rho \cos \rho-\sin \rho)^{2}\left[\mathbf{e}_{\mathbf{r}} \sin \vartheta \cos \varphi\left(2-2 i k_{\mathrm{pl}} r-k_{\mathrm{pl}}^{2} r^{2}\right)+\left(\mathbf{e}_{\vartheta} \cos \vartheta \cos \varphi-\mathbf{e}_{\varphi} \sin \varphi\right)\left(-1+i k_{\mathrm{pl}} r\right)\right]\right\}
\end{aligned}
$$


For $\mathbf{e}_{\mathrm{mol}}=\boldsymbol{\varepsilon}_{\mathrm{pt}}=\mathbf{e}_{x}$ and $\mathbf{k}_{\mathrm{pt}}=k_{\mathrm{pl}} \mathbf{e}_{z}$ we obtain

$$
\begin{aligned}
\beta(r, \vartheta, \varphi)= & \frac{9}{8} \frac{1+\rho^{2}-\rho^{4}+\left(-1+\rho^{2}\right) \cos (2 \rho)+\rho\left(-2+\rho^{2} / 2\right) \sin (2 \rho)}{\rho^{6}(\rho \cos \rho-\sin \rho)^{2}} \frac{e^{i k_{\mathrm{pl}} r(1-\cos \vartheta)}}{k_{\mathrm{pl}}^{3} r^{3}}\left\{k_{\mathrm{pl}}^{2} r^{2} \rho^{3}[-2 \rho+\sin (2 \rho)] / 6\right. \\
& +\left[-6-6 \rho^{2}+2 \rho^{4}+\left(6-6 \rho^{2}\right) \cos (2 \rho)-\rho\left(-12+\rho^{2}\right) \sin (2 \rho)\right]\left(-3+3 i k_{\mathrm{pl}} r+k_{\mathrm{pl}}^{2} r^{2}\right) \\
& \times\left(2 \sin ^{2} \vartheta \cos ^{2} \varphi-\cos ^{2} \vartheta \cos ^{2} \varphi-\sin ^{2} \varphi\right) / 12-2(\rho \cos \rho-\sin \rho)^{2} \\
& \left.\times\left[\sin ^{2} \vartheta \cos ^{2} \varphi\left(2-2 i k_{\mathrm{pl}} r-k_{\mathrm{pl}}^{2} r^{2}\right)+\left(\cos ^{2} \vartheta \cos ^{2} \varphi+\sin ^{2} \varphi\right)\left(-1+i k_{\mathrm{p} 1} r\right)\right]\right\}
\end{aligned}
$$

in Eq. (31). We abbreviated $\rho=k_{\mathrm{pl}} a$.

Within the quasistatic point-dipole approximation, i.e., Eq. (30), we find $\varepsilon_{\mathrm{pl}}^{\mathrm{pt}}=1 / \sqrt{3}$ and

$$
\begin{aligned}
\mathbf{G}_{\mathrm{pl}}(\mathbf{r}) & =\frac{1}{\sqrt{3}} \stackrel{\leftrightarrow}{\mathbf{G}}(\mathbf{r}, 0) \mathbf{e}_{x}=\frac{1}{\sqrt{3}} G_{0}(\mathbf{r}, 0) \mathbf{e}_{x}+\frac{1}{\sqrt{3} k_{\mathrm{pl}}^{2}} \nabla_{\mathbf{r}}\left\{\nabla_{\mathbf{r}} \cdot\left[G_{0}(\mathbf{r}, 0) \mathbf{e}_{x}\right]\right\} \\
& =\frac{k_{\mathrm{p} 1} e^{i k_{\mathrm{p} 1} r}}{4 \sqrt{3} \pi}\left[\mathbf{e}_{\mathbf{r}} \sin \vartheta \cos \varphi \frac{2-2 i k_{\mathrm{pl}} r}{k_{\mathrm{pl}}^{3} r^{3}}+\left(\mathbf{e}_{\vartheta} \cos \vartheta \cos \varphi-\mathbf{e}_{\varphi} \sin \varphi\right) \frac{k_{\mathrm{pl}}^{2} r^{2}+i k_{\mathrm{p} 1} r-1}{k_{\mathrm{pl}}^{3} r^{3}}\right],
\end{aligned}
$$

which leads to

$$
\beta(r, \vartheta, \varphi)=\frac{e^{i k_{\mathrm{pl}} r(1-\cos \vartheta)}}{k_{\mathrm{pl}}^{3} r^{3}}\left[\sin ^{2} \vartheta \cos ^{2} \varphi\left(2-2 i k_{\mathrm{pl}} r\right)+\left(\cos ^{2} \vartheta \cos ^{2} \varphi+\sin ^{2} \varphi\right)\left(k_{\mathrm{pl}}^{2} r^{2}+i k_{\mathrm{p} 1} r-1\right)\right]
$$

in Eq. (31).

\section{APPENDIX C: EM ENHANCEMENT}

The EM enhancement is calculated as

$$
\operatorname{EF}_{\mathrm{EM}}\left(\omega_{\mathrm{L}}\right)=\frac{\left|\mathbf{E}_{\text {inc }}+\mathbf{E}_{\mathrm{LSP}}\left(\omega_{\mathrm{L}}\right)\right|^{2}}{\left|\mathbf{E}_{\text {inc }}\right|^{2}} \frac{\left|\mathbf{E}_{\text {inc }}+\mathbf{E}_{\mathrm{LSP}}\left(\omega_{\mathrm{S}}\right)\right|^{2}}{\left|\mathbf{E}_{\text {inc }}\right|^{2}},
$$

with the incident electric field $\mathbf{E}_{\text {inc }}$ and the electric field generated by the plasmonic nanostructure $\mathbf{E}_{\mathrm{LSP}}$ at the frequency $\omega_{\mathrm{L}}$ of the incoming laser or the frequency $\omega_{\mathrm{S}}$ of the Raman scattered light. This expression is a good approximation of the EM enhancement in the backscattering configuration, which is considered in this article [4,9]. We also calculate the EM enhancement with the EM-DR following the steps in Ref. [8].

Beside a single gold nanosphere we use $\mathbf{E}_{\mathrm{LSP}}=\mathbf{E}_{\mathrm{sca}}$ from Eq. (A4) within Mie theory and

$$
\mathbf{E}_{\mathrm{LSP}}(\mathbf{r}, \omega)=\frac{k^{2} \alpha(\omega)}{\epsilon_{0} \epsilon_{\mathrm{m}}} E_{0} \stackrel{\leftrightarrow}{\mathbf{G}}(\mathbf{r}, 0) \mathbf{e}_{x}
$$

within the quasistatic approximation (for $\mathbf{E}_{\mathrm{inc}}=E_{0} \mathbf{e}_{x}$ );

$$
\alpha(\omega)=4 \pi \epsilon_{0} \epsilon_{\mathrm{m}} a^{3} \frac{\epsilon_{\mathrm{Au}}(\omega)-\epsilon_{\mathrm{m}}}{\epsilon_{\mathrm{Au}}(\omega)+2 \epsilon_{\mathrm{m}}}
$$

is the polarizability of the gold nanosphere. We use an analytical model for the dielectric function of gold $\epsilon_{\mathrm{Au}}(\omega)$ from Ref. [55] to reproduce the experimental data from Ref. [56].

We calculate the enhancement in the hot spot of a gold nanosphere dimer within the quasistatic point-dipole approximation following the steps in Ref. [57]. The dimer consists of two gold spheres with radius $a$ and center-to-center distance $d$. The spheres are placed on the $x$ axis at $\mathbf{d} / 2$ (sphere 1) and $-\mathbf{d} / 2$ (sphere 2). For light polarization along the dimer axis, the local electric field acting on sphere 1 is

$$
\begin{aligned}
\mathbf{E}_{1}^{\mathrm{loc}} & =\mathbf{E}_{\mathrm{inc}}+\mathbf{E}_{2}^{\mathrm{pl}}(d / 2,0,0) \\
& =\mathbf{E}_{\text {inc }}+\frac{\alpha(\omega) e^{i k_{\mathrm{pl}} d}}{2 \pi \epsilon_{0} \epsilon_{m} d^{3}}\left(1-i k_{\mathrm{pl}} d\right) \mathbf{E}_{2}^{\mathrm{loc}},
\end{aligned}
$$

where $\mathbf{E}_{2}^{\mathrm{pl}}(d / 2,0,0)$ is the scattered field of sphere 2 at the position of sphere 1 [calculated with Eqs. (C2) and (B10)]. Similarly, the local electric field acting on sphere 2 is

$$
\begin{aligned}
\mathbf{E}_{2}^{\mathrm{loc}} & =\mathbf{E}_{\mathrm{inc}}+\mathbf{E}_{1}^{\mathrm{pl}}(-d / 2,0,0) \\
& =\mathbf{E}_{\text {inc }}+\frac{\alpha(\omega) e^{i k_{\mathrm{pl}} d}}{2 \pi \epsilon_{0} \epsilon_{m} d^{3}}\left(1-i k_{\mathrm{pl}} d\right) \mathbf{E}_{1}^{\mathrm{loc}} .
\end{aligned}
$$

This system of two coupled equations can be solved for $\mathbf{E}_{1,2}^{\text {loc }}$, which leads to

$$
\mathbf{E}_{1}^{\mathrm{loc}}=\mathbf{E}_{2}^{\mathrm{loc}}=\frac{1+\frac{\alpha(\omega)^{i k_{\mathrm{pl}} d}}{2 \pi \epsilon_{m} d^{3}}\left(1-i k_{\mathrm{pl}} d\right)}{1-\left[\frac{\alpha(\omega) e^{i k_{\mathrm{p}} d}}{2 \pi \epsilon_{0} \epsilon_{m} d^{3}}\left(1-i k_{\mathrm{pl}} d\right)\right]^{2}} \mathbf{E}_{\mathrm{inc}} .
$$

The induced dipole moment in the nanosphere dimer is

$$
\mathbf{p}(\mathbf{r}, \omega)=\alpha_{\|}^{\text {ind }}(\omega) \mathbf{E}_{\text {inc }}[\delta(\mathbf{r}-\mathbf{d} / 2)+\delta(\mathbf{r}+\mathbf{d} / 2)],
$$

with

$$
\alpha_{\|}^{\text {ind }}=\alpha(\omega) \frac{1+\frac{\alpha(\omega) e^{i k_{\mathrm{p}} d}}{2 \pi \epsilon_{0} \epsilon_{m} d^{3}}\left(1-i k_{\mathrm{pl}} d\right)}{1-\left[\frac{\alpha(\omega) e^{i k_{\mathrm{p}} d} d}{2 \pi \epsilon_{0} \epsilon_{m} d^{3}}\left(1-i k_{\mathrm{pl}} d\right)\right]^{2}} .
$$

The electric field generated by the nanosphere dimer is then

$$
\mathbf{E}_{\mathrm{LSP}}(\mathbf{r}, \omega)=\frac{k^{2} \alpha_{\|}^{\mathrm{ind}}(\omega)}{\epsilon_{0} \epsilon_{\mathrm{m}}} E_{0}[\stackrel{\leftrightarrow}{\mathbf{G}}(\mathbf{r},-\mathbf{d} / 2)+\stackrel{\leftrightarrow}{\mathbf{G}}(\mathbf{r}, \mathbf{d} / 2)] \mathbf{e}_{x} .
$$

For light polarization perpendicular to the dimer axis we obtain

$$
\alpha_{\perp}^{\text {ind }}=\alpha(\omega) \frac{1-\frac{\alpha(\omega) e^{i k_{\mathrm{p}} d}}{4 \pi \epsilon_{0} \epsilon_{m} d^{3}}\left(1-i k_{\mathrm{pl}} d-k_{\mathrm{pl}}^{2} d^{2}\right)}{1-\left[\frac{\alpha(\omega) e_{\mathrm{p}} d^{2}}{4 \pi \epsilon_{0} \epsilon_{m} d^{3}}\left(1-i k_{\mathrm{pl}} d-k_{\mathrm{pl}}^{2} d^{2}\right)\right]^{2}} .
$$


(a)

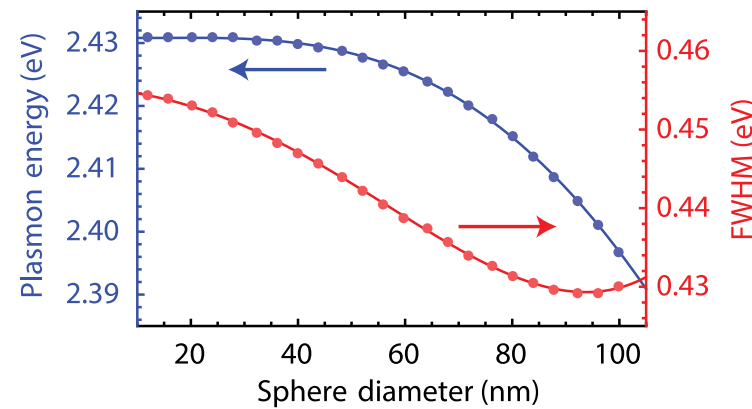

(b)

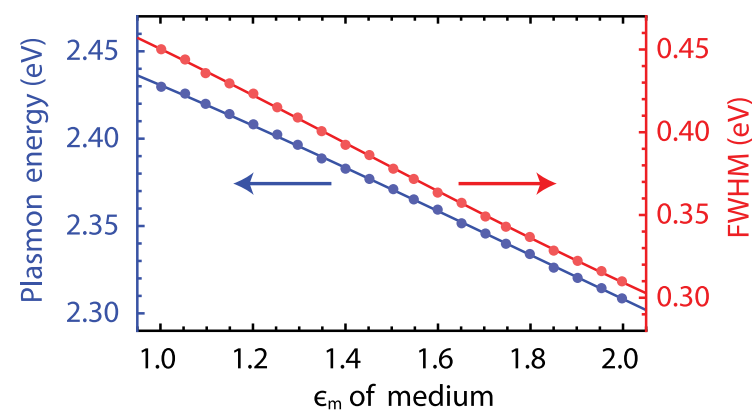

FIG. 6. Plasmon energy $\hbar \omega_{\mathrm{pl}}$ (blue) and full width at half maximum $2 \gamma_{\mathrm{pl}}$ (red) as function of (a) sphere diameter and (b) dielectric constant $\epsilon_{\mathrm{m}}$ of surrounding medium. Data represented by plot markers were obtained from fit of $\left|d_{1}(\omega)\right|^{2}$, Eq. (A13). Solid lines are fit of these data with polynomial.

\section{APPENDIX D: ENERGY AND SPECTRAL WIDTH OF PLASMON}

In this Appendix we describe, how we obtained the energy $\hbar \omega_{\mathrm{pl}}$ and spectral width $2 \gamma_{\mathrm{pl}}$ of the dipole LSP mode for the plots in Figs. 3, 4, and 5.

For the calculation of the SERS enhancement beside a single $\mathrm{Au}$ nanosphere, i.e., Figs. 3 and 4 , we deduced $\omega_{\mathrm{pl}}$ and $\gamma_{\mathrm{pl}}$ from the excitation-energy dependence of the internal electric field given by $\left|d_{n=1}(\omega)\right|^{2}$. Values for $\omega_{\mathrm{pl}}$ and $\gamma_{\mathrm{pl}}$ were obtained from a fit with a single Lorentzian peak for several sphere diameters and dielectric constants $\epsilon_{\mathrm{m}}$ of the surrounding medium [plot markers in Figs. 6(a) and 6(b)]. These values were then fit with a polynomial to obtain $\omega_{\mathrm{pl}}$ and $\gamma_{\mathrm{pl}}$ as a function of sphere diameter and $\epsilon_{\mathrm{m}}$ [lines in Figs. 6(a)
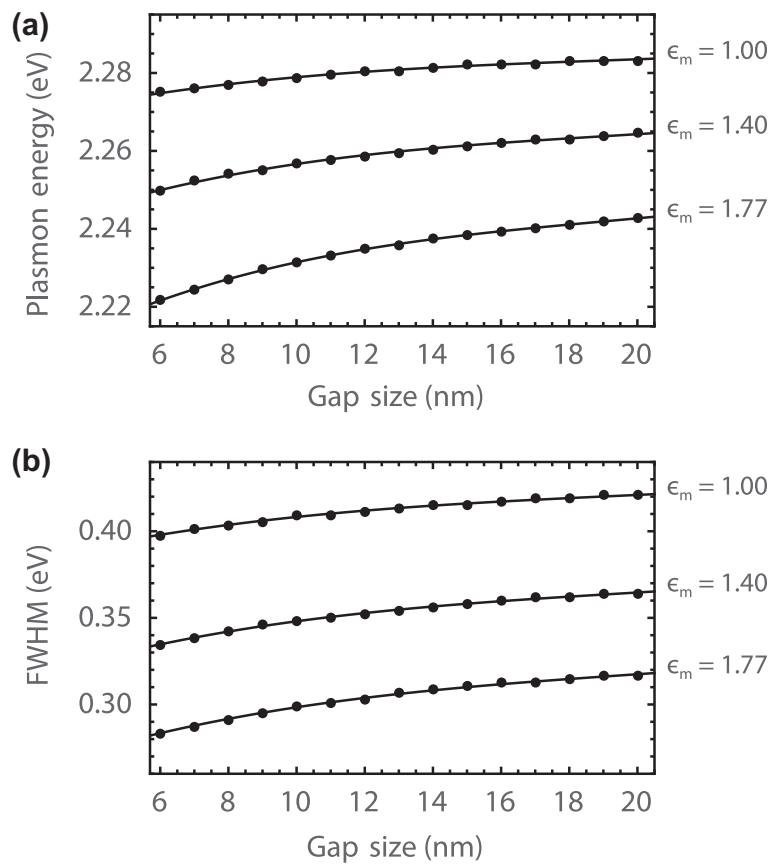

FIG. 7. (a) Plasmon energy $E_{\mathrm{pl}}=\hbar \omega_{\mathrm{pl}}$ and (b) spectral width $\mathrm{FWHM}=2 \gamma_{\mathrm{pl}}$ as function of gap size of nanosphere dimer for three dielectric constants $\epsilon_{\mathrm{m}}$. Data represented by plot markers were obtained from a fit of $\left|\alpha_{\|}^{\text {ind }}(\omega, d)\right|^{2}$, Eq. (C8). Solid lines are fit of these data with polynomial.

and 6(b)]. For the calculation of the enhancement within the quasistatic approximation in Fig. 3(a), we used the values of $\omega_{\mathrm{pl}}$ and $\gamma_{\mathrm{pl}}$ for the smallest sphere diameter of $10 \mathrm{~nm}$ in Fig. 6(a).

To calculate the SERS enhancement in the center of the gold nanosphere dimer, we obtained $\omega_{\mathrm{pl}}$ and $\gamma_{\mathrm{pl}}$ from a fit of the dimer polarizability $\left|\alpha_{\|}^{\text {ind }}(\omega, d)\right|^{2}$, Eq. (C8), with a single Lorentzian peak and a constant background. Values of $\omega_{\mathrm{pl}}$ and $\gamma_{\mathrm{pl}}$ were calculated as a function of gap size $d$ (in steps of $1 \mathrm{~nm}$ ) for three values of $\epsilon_{\mathrm{m}}$ [plot markers in Figs. 7(a) and 7(b)]. These discrete values were fit with a polynomial to obtain the functions $\omega_{\mathrm{pl}}\left(d, \epsilon_{\mathrm{m}}\right)$ and $\gamma_{\mathrm{pl}}\left(d, \epsilon_{\mathrm{m}}\right)$; see solid lines in Fig. 7. A similar approach was used to determine the plasmon energies $\omega_{\mathrm{pl}, \perp}$ in Eq. (34) from $\left|\alpha_{\perp}^{\text {ind }}(\omega, d)\right|^{2}$.
[1] M. Fleischmann, P. Hendra, and A. McQuillan, Chem. Phys. Lett. 26, 163 (1974).

[2] S. Nie and S. R. Emory, Science 275, 1102 (1997).

[3] K. Kneipp, Y. Wang, H. Kneipp, L. T. Perelman, I. Itzkan, R. R. Dasari, and M. S. Feld, Phys. Rev. Lett. 78, 1667 (1997).

[4] E. C. L. Ru and P. G. Etchegoin, Principles of Surface-enhanced Raman Spectroscopy (Elsevier, Amsterdam, 2009).

[5] B. Sharma, R. R. Frontiera, A.-I. Henry, E. Ringe, and R. P. V. Duyne, Mater. Today 15, 16 (2012).

[6] M. Moskovits, Rev. Mod. Phys. 57, 783 (1985).

[7] S. Maier, Plasmonics: Fundamentals and Applications (Springer, New York, 2007).
[8] L. K. Ausman and G. C. Schatz, J. Chem. Phys. 131, 084708 (2009).

[9] L. Ausman and G. Schatz, in The Mie Theory, edited by W. Hergert and T. Wriedt, Springer Series in Optical Sciences Vol. 169 (Springer, Berlin Heidelberg, 2012), pp. 135-155.

[10] K. L. Wustholz, A.-I. Henry, J. M. McMahon, R. G. Freeman, N. Valley, M. E. Piotti, M. J. Natan, G. C. Schatz, and R. P. V. Duyne, J. Am. Chem. Soc. 132, 10903 (2010).

[11] W. Zhu and K. B. Crozier, Nat. Commun. 5, 5228 (2014).

[12] W. Zhu, M. G. Banaee, D. Wang, Y. Chu, and K. B. Crozier, Small 7, 1761 (2011).

[13] D. Talaga, M. Comesaa-Hermo, S. Ravaine, R. A. L. Valle, and S. Bonhommeau, J. Opt. 17, 114006 (2015). 
[14] D.-K. Lim, K.-S. Jeon, J.-H. Hwang, H. Kim, S. Kwon, Y. D. Suh, and J.-M. Nam, Nat. Nano. 6, 452 (2011).

[15] J. R. Lombardi and R. L. Birke, J. Chem. Phys. 136, 144704 (2012).

[16] M. Thomas, S. Mhlig, T. Deckert-Gaudig, C. Rockstuhl, V. Deckert, and P. Marquetand, J. Raman Spectrosc. 44, 1497 (2013).

[17] J. L. Payton, S. M. Morton, J. E. Moore, and L. Jensen, J. Chem. Phys. 136, 214103 (2012).

[18] J. L. Payton, S. M. Morton, J. E. Moore, and L. Jensen, Acc. Chem. Res. 47, 88 (2014).

[19] D. J. Masiello and G. C. Schatz, Phys. Rev. A 78, 042505 (2008).

[20] D. J. Masiello and G. C. Schatz, J. Chem. Phys. 132, 064102 (2010).

[21] J. P. Litz, R. P. Brewster, A. B. Lee, and D. J. Masiello, J. Phys. Chem. C 117, 12249 (2013).

[22] A. M. Kelley, J. Chem. Phys. 128, 224702 (2008).

[23] P. Johansson, H. Xu, and M. Käll, Phys. Rev. B 72, 035427 (2005).

[24] V. N. Pustovit and T. V. Shahbazyan, Phys. Rev. B 73, 085408 (2006).

[25] W. Gu, H. Choi, and K. K. Kim, J. Phys. Chem. A 111, 8121 (2007).

[26] M. Galperin, M. A. Ratner, and A. Nitzan, J. Chem. Phys. 130, 144109 (2009).

[27] P. Roelli, C. Galland, N. Piro, and T. J. Kippenberg, Nat. Nanotechnol. 11, 164 (2016).

[28] D. A. Long, The Raman Effect (Wiley \& Sons, New York, 2002).

[29] P. Y. Yu and M. Cardona, Fundamentals of Semiconductors: Physics and Materials Properties, 4th ed. (Springer, Berlin, 2010).

[30] A. M. Kelley, Annu. Rev. Phys. Chem. 61, 41 (2010).

[31] S. Nie, L. A. Lipscomb, and N.-T. Yu, Appl. Spectrosc. Rev. 26, 203 (1991).

[32] S. Dey, M. Banik, E. Hulkko, K. Rodriguez, V. A. Apkarian, M. Galperin, and A. Nitzan, Phys. Rev. B 93, 035411 (2016).

[33] G. Abstreiter, M. Cardona, and A. Pinczuk, in Light Scattering in Solids IV, edited by M. Cardona and G. Güntherodt, Topics in Applied Physics Vol. 54 (Springer, Berlin, Heidelberg, 1984), pp. $5-150$.

[34] J. R. Lombardi and R. L. Birke, J. Phys. Chem. C 112, 5605 (2008).

[35] E. J. Ayars, H. D. Hallen, and C. L. Jahncke, Phys. Rev. Lett. 85, 4180 (2000).

[36] M. Finazzi and F. Ciccacci, Phys. Rev. B 86, 035428 (2012).
[37] A. Manjavacas, F. J. G. de Abajo, and P. Nordlander, Nano Lett. 11, 2318 (2011).

[38] R. Loudon, The Quantum Theory of Light, 3rd ed. (Oxford University Press, Oxford, 2000).

[39] S.-T. Ho and P. Kumar, J. Opt. Soc. Am. B 10, 1620 (1993).

[40] E. Waks and D. Sridharan, Phys. Rev. A 82, 043845 (2010).

[41] L. Novotny and B. Hecht, Principles of Nano-optics, 2nd ed. (Cambridge University Press, Cambridge, U.K., 2012).

[42] S.-O. Guillaume, F. J. Garcia de Abajo, and L. Henrard, Phys. Rev. B 88, 245439 (2013).

[43] U. Hohenester and J. Krenn, Phys. Rev. B 72, 195429 (2005).

[44] C. Sönnichsen, T. Franzl, T. Wilk, G. von Plessen, J. Feldmann, O. Wilson, and P. Mulvaney, Phys. Rev. Lett. 88, 077402 (2002).

[45] The effect of a substrate on a plasmonic nanostructure can be estimated by an effective dielectric constant $\epsilon_{\mathrm{m}, \text { eff }}$. For details, see, e.g., Ref. [46].

[46] W. Knoben, S. H. Brongersma, and M. Crego-Calama, Nanotech. 22, 295303 (2011).

[47] J. P. Camden, J. A. Dieringer, Y. Wang, D. J. Masiello, L. D. Marks, G. C. Schatz, and R. P. Van Duyne, J. Am. Chem. Soc. 130, 12616 (2008).

[48] Even though the point-dipole approximation is not applicable for $d<3 a$, we expect the difference between enhancement from our approach and EM enhancement to be estimated correctly here. The point-dipole approximation will lead to the same deviations in both theories.

[49] S. L. Kleinman, B. Sharma, M. G. Blaber, A.-I. Henry, N. Valley, R. G. Freeman, M. J. Natan, G. C. Schatz, and R. P. V. Duyne, J. Am. Chem. Soc. 135, 301 (2013).

[50] L. Jensen, L. L. Zhao, J. Autschbach, and G. C. Schatz, J. Chem. Phys. 123, 174110 (2005).

[51] S. Heeg, R. Fernandez-Garcia, A. Oikonomou, F. Schedin, R. Narula, S. A. Maier, A. Vijayaraghavan, and S. Reich, Nano Lett. 13, 301 (2013)

[52] F. Schedin, E. Lidorikis, A. Lombardo, V. G. Kravets, A. K. Geim, A. N. Grigorenko, K. S. Novoselov, and A. C. Ferrari, ACS Nano 4, 5617 (2010).

[53] C. F. Bohren and D. R. Huffman, Absorption and Scattering of Light by Small Particles (Wiley \& Sons, New York, 1983).

[54] J. D. Jackson, Classical Electrodynamics, 3rd ed. (Wiley, New York, 1999).

[55] P. G. Etchegoin, E. C. Le Ru, and M. Meyer, J. Chem. Phys. 125, 164705 (2006).

[56] P. B. Johnson and R. W. Christy, Phys. Rev. B 6, 4370 (1972).

[57] A. Pinchuk and G. Schatz, Nanotechnology 16, 2209 (2005). 